\title{
ON THE CONTINUATION OF AN INVARIANT TORUS IN A FAMILY WITH RAPID OSCILLATIONS*
}

\author{
CARMEN CHICONE ${ }^{\dagger}$ AND WEISHI LIU ${ }^{\dagger}$ \\ Dedicated to Professor Jack Hale on the occasion of his seventieth birthday.
}

\begin{abstract}
A persistence theorem for attracting invariant tori for systems subjected to rapidly oscillating perturbations is proved. The singular nature of these perturbations prevents the direct application of the standard persistence results for normally hyperbolic invariant manifolds. However, as is illustrated in this paper, the theory of normally hyperbolic invariant manifolds, when combined with an appropriate continuation method, does apply.
\end{abstract}

Key words. persistence, continuation, rapid oscillation, invariant torus

AMS subject classifications. 34C15, 58F 30

PII. S0036141098338740

1. Introduction. We will prove a persistence theorem for attracting invariant tori for systems subjected to rapidly oscillating perturbations. The singular nature of these perturbations prevents the direct application of the standard persistence results for normally hyperbolic invariant manifolds. However, as we will illustrate in this paper, the theory of normally hyperbolic invariant manifolds, when combined with an appropriate continuation method, does apply.

Systems with rapidly oscillating perturbations arise naturally when a priori stable systems are periodically forced. In fact, partial averaging (perhaps to some high order) at a resonant torus together with a rescaling to slow time produces a system with a rapidly oscillating perturbation. For example, systems of this type are obtained in the dissipative periodically forced oscillator models introduced in [2].

We will consider the existence of invariant tori for a smooth family of differential equations of the form

$$
\dot{x}=f(x, \epsilon)+\epsilon g(x, t, \epsilon),
$$

where $g$ is a periodic function of the independent variable. For systems of this type, the behavior of the subsystem

$$
\dot{x}=f(x, \epsilon)
$$

at $\epsilon=0$ is important. If this subsystem has a normally hyperbolic invariant manifold at $\epsilon=0$, then the persistence of this manifold into the full system is a result of the general persistence theorems of Fenichel [3] and Hirsch, Pugh, and Shub [7]. However, in many applications, either there is an invariant manifold at $\epsilon=0$ that is not normally hyperbolic or the system is singular at $\epsilon=0$. For example, the first situation will arise when a periodically forced oscillator is averaged at a resonance. In these cases,

\footnotetext{
*Received by the editors May 14, 1998; accepted for publication (in revised form) May 18, 1999; published electronically January $5,2000$.

http://www.siam.org/journals/sima/31-2/33874.html

${ }^{\dagger}$ Department of Mathematics, University of Missouri, Columbia, MO 65211 (carmen@chicone. math.missouri.edu, liu@math.missouri.edu). The research of the first author was supported by the National Science Foundation under grant DMS-9303767.
} 
a change of coordinates and a rescaling of the independent variable often yields an equivalent family, for $\epsilon \neq 0$, of the form

$$
y^{\prime}=F(y)+\epsilon G(y, \tau / \epsilon, \epsilon)
$$

with new independent variable $\tau$. While the subsystem

$$
y^{\prime}=F(y)
$$

of the family (1.1) in these new coordinates no longer depends on the perturbation parameter, and is therefore regular, the singular nature of the perturbation is reflected in system (1.1) by rapid oscillations of the perturbation term in the slow time.

If the subsystem (1.2) has a normally hyperbolic invariant torus, then a small $C^{1}$ perturbation will also have a normally hyperbolic invariant torus by the general persistence theorems mentioned above. However, in the full system (1.1), the perturbation is not defined at $\epsilon=0$. Also, we note that the perturbation is not $C^{1}$ small. In fact, the partial derivative with respect to $\tau$ can be large relative to $\epsilon$. Thus, the usual persistence theory does not apply directly. To overcome this difficulty we will use the idea introduced by Kopell [8] of embedding the system into an auxiliary family given by

$$
y^{\prime}=F(y)+\delta G(y, \tau / \epsilon, \epsilon) .
$$

If $\epsilon>0$ is fixed and $\delta>0$ is sufficiently small, then, by the usual persistence theory, the system (1.3) has a normally hyperbolic invariant manifold. We will show that if $\epsilon>0$ is sufficiently small, then this normally hyperbolic invariant manifold can be continued in this family to $\delta=\epsilon$.

The plan of the paper is as follows. In section 2 , a description of the origin of the model system that we will study is given. In section 3 we discuss some previous work by Kopell [8] on the continuation problem for system (1.3). A conceptual gap in this work will be described. Also, we will present the general method for continuation that is used in this paper. The statement of our main theorem on the existence of invariant tori is in section 4 , and the remaining sections of the paper are devoted to its proof.

2. A periodically perturbed oscillator. In this section we will briefly describe the origin of the explicit perturbation problem that we will study; see [2] for more details.

Consider a periodically perturbed planar oscillator given by

$$
\dot{u}=f(u)+\epsilon g(u, t),
$$

where, for each $u \in \mathbb{R}^{2}$, the function $t \mapsto g(u, t)$ is $2 \pi / \Omega$ periodic and $\epsilon$ is a small parameter. Let us assume that the unperturbed system

$$
\dot{u}=f(u)
$$

is Hamiltonian and it has a regular period annulus $\mathcal{A}$, that is, an annulus consisting entirely of periodic orbits such that the associated period function is regular. Also, for each point $\zeta$ in the domain of definition of the system $(2.1)$, let $t \mapsto u(t, \zeta, \epsilon)$ denote the solution of (2.1) with the initial condition $u(0, \zeta, \epsilon)=\zeta$. 
A periodic orbit $\Gamma$ in $\mathcal{A}$ with period $T$ is called resonant if there are relatively prime positive integers $m$ and $n$ such that

$$
m \frac{2 \pi}{\Omega}=n T .
$$

If $\Gamma$ is a resonant periodic orbit and $p \in \Gamma$, then the associated (subharmonic) Melnikov function is given by

$$
M^{m: n}(\phi):=\int_{0}^{m 2 \pi / \Omega} f(u(t, p, 0)) \wedge g(u(t, p, 0), t-\phi) d t .
$$

From a geometric point of view, the sign of the Melnikov function on a resonant orbit determines the "drift direction" for perturbed orbits. If, for example, the Melnikov function has a fixed sign, then perturbed orbits drift away from the vicinity of the resonant orbit in a direction determined by this sign; while if the Melnikov function has a simple zero, then there is a nearby perturbed periodic orbit; see [5], [9], [11].

If the Melnikov function vanishes identically on a resonant orbit, then a reasonable expectation is that the corresponding unperturbed torus in the phase cylinder, corresponding to the unperturbed resonant orbit, persists under the perturbation. If the perturbation is dissipative, then the perturbed invariant torus is an attractor. The presence of this attractor is often one of the dominant features of the global dynamics: perturbed orbits are entrained to this torus. Thus, the existence of invariant tori is an important consideration in the analysis of the global dynamics of the system. However, as we will see, the proof of the existence of an attracting invariant torus in this context requires additional hypotheses as well as a delicate perturbation analysis.

To study the dynamics of differential (2.1) near a resonant periodic orbit, it is convenient to consider the system in action angle coordinates. In fact, there is a smooth change of coordinates in a neighborhood of the resonant orbit such that the differential $(2.1)$, in the new coordinates $(I, \vartheta)$, has the form

$$
\dot{I}=\epsilon F(I, \vartheta, t), \quad \dot{\vartheta}=\omega(I)+\epsilon G(I, \vartheta, t),
$$

where both $F$ and $G$ are $2 \pi$ periodic in $\vartheta$ and $2 \pi m / \Omega$ periodic in $t$. In these coordinates, the resonant orbit is given by $\left\{(I, \vartheta): I=I_{0}\right\}$, where

$$
m \frac{2 \pi}{\Omega}=n \frac{2 \pi}{\omega\left(I_{0}\right)} .
$$

A "normal form" for system (2.5) with $\epsilon>0$ at the resonant orbit is obtained by using the coordinate transformation

$$
I=I_{0}+\sqrt{\epsilon} \ell, \quad \vartheta=\omega\left(I_{0}\right) t+\sigma,
$$

followed by the Taylor expansion of the resulting vector field to third order in powers of $\sqrt{\epsilon}$. The transformed system

$$
\begin{aligned}
\dot{\ell}= & \sqrt{\epsilon} F\left(I_{0}, \omega\left(I_{0}\right) t+\sigma, t\right)+\epsilon F_{I}\left(I_{0}, \omega\left(I_{0}\right) t+\sigma, t\right) \ell \\
& +\epsilon^{3 / 2} F_{I I}\left(I_{0}, \omega\left(I_{0}\right) t+\sigma, t\right) \ell^{2}+O\left(\epsilon^{2}\right) \\
\dot{\sigma}= & \sqrt{\epsilon} \omega^{\prime}\left(I_{0}\right) \ell+\epsilon\left(G\left(I_{0}, \omega\left(I_{0}\right) t+\sigma, t\right)+\frac{1}{2} \omega^{\prime \prime}\left(I_{0}\right) \ell^{2}\right) \\
& +\epsilon^{3 / 2}\left(G_{I}\left(I_{0}, \omega\left(I_{0}\right) t+\sigma, t\right) \ell+\frac{1}{6} \omega^{\prime \prime \prime}\left(I_{0}\right) \ell^{3}\right)+O\left(\epsilon^{2}\right)
\end{aligned}
$$


is in the "time periodic standard form," the correct form for averaging. Under the assumption that the Melnikov function vanishes on the resonant orbit, that is, the average of $F$ in the new coordinates vanishes, there is an averaging transformation (slightly more general than the transformation used in [2] where the average of $G$ is also assumed to vanish) such that the averaged system has the abstract form

$$
\begin{aligned}
\dot{\ell} & =\mu^{2} p(\sigma) \ell+\mu^{3}\left(q(\sigma) \ell^{2}+r(\sigma)\right)+\mu^{4} \widehat{R}(\ell, \sigma, t, \mu), \\
\dot{\sigma} & =\mu \lambda \ell+\mu^{2}\left(\nu \ell^{2}+g(\sigma)\right)+\mu^{3} \widehat{S}(\ell, \sigma, t, \mu),
\end{aligned}
$$

where $p, q, r$, and $g$ are $2 \pi$ periodic functions, $\lambda, \mu$, and $\nu$ are real numbers, and both of the functions $\widehat{R}$ and $\widehat{S}$ are $2 \pi$ periodic in $\sigma$ and $2 \pi / \Omega$ periodic in $t$. In fact, all of the functions appearing in the system (2.8) are identifiable in terms of the original vector field. Also, the new small parameter is defined by $\mu:=\sqrt{\epsilon}$.

Let us rewrite system (2.8) as the autonomous system

$$
\begin{aligned}
\dot{\ell} & =\mu^{2} p(\sigma) \ell+\mu^{3}\left(q(\sigma) \ell^{2}+r(\sigma)\right)+\mu^{4} \widehat{R}(\ell, \sigma, \varphi, \mu), \\
\dot{\sigma} & =\mu \lambda \ell+\mu^{2}\left(\nu \ell^{2}+g(\sigma)\right)+\mu^{3} \widehat{S}(\ell, \sigma, \varphi, \mu), \\
\dot{\varphi} & =1
\end{aligned}
$$

where $\varphi$ is a new angular variable modulo $2 \pi m / \Omega$. Also, let us assume that the family is class $C^{\infty}$. We will seek an invariant torus for system (2.9) as the graph of a periodic function $(\sigma, \varphi) \mapsto h(\sigma, \varphi)$; that is, $h$ is $2 \pi$ periodic in $\sigma$ and $2 \pi m / \Omega$ periodic in $\varphi$.

The Lyapunov-Perron method is used in [2] to prove the following theorem.

Theorem 2.1. Consider the differential (2.9) and define

$$
M:=\min _{0 \leq \sigma \leq 2 \pi}|p(\sigma)|>0
$$

If $g(\sigma) \equiv 0, \lambda \neq 0$,

$$
5 M>\operatorname{Lip}(p), \quad M^{2} \geq 6|\lambda|\|r\|_{0,1},
$$

and $\mu$ is sufficiently small, then there is a periodic function $h \in C^{0,1}$ (supremum + Lipschitz norm) such that its graph $\{(\ell, \sigma, t): \ell=h(\sigma, t)\}$ is an invariant torus for (2.9).

Here, $M$ is a measure of the minimum "normal contraction rate" and the inequality $M^{2} \geq 6|\lambda|\|r\|_{0,1}$ is a sufficient condition to preclude "roll up" of the invariant manifold at a sink; see the example in $[2$, p. 63]. The inequality $5 M>\operatorname{Lip}(p)$ does not seem to have a geometric interpretation; rather it arises from the technical estimates in the proof.

We note that Robinson and Murdock in [10] prove the existence of invariant tori for a differential equation similar to system (2.9). Their result concerns the continuation of certain nonresonant unperturbed tori in analytic systems.

3. Normal hyperbolicity and continuation. In this section we recall the definition of normal hyperbolicity and discuss the basic idea, introduced by Kopell [8], that we will use to continue invariant manifolds. While our continuation method applies to normally hyperbolic invariant manifolds with expanding and contracting normal directions, in this paper we discuss only the case of normally hyperbolic invariant manifolds with no unstable normal directions. In particular, when we use the phrase "normally hyperbolic" we will use it in this restricted sense. 
Let us consider a smooth differential equation

$$
\dot{x}=F(x), \quad x \in \mathbb{R}^{n}
$$

with flow $\phi^{t}$ that has an overflowing invariant manifold $\bar{M}=M \cup \partial M$. Also, let $T M$ denote the tangent bundle of $M$, and, with respect to the usual inner product on $\mathbb{R}^{n}$, let $N$ denote the bundle normal to $T M$ over $M$. Then,

$$
T_{M} \mathbb{R}^{n}=T M \oplus N
$$

and there is a natural orthogonal projection $\Pi: T_{M} \mathbb{R}^{n} \rightarrow N$. Recall that in this context (see [3]) there are operators

$$
\begin{aligned}
& A_{t}(p):=\left.D \phi^{-t}(p)\right|_{T_{p} M}: T_{p} M \rightarrow T_{\phi^{-t}(p)} M, \\
& B_{t}(p):=\left.\Pi_{p} D \phi^{t}\left(\phi^{-t}(p)\right)\right|_{N_{\phi^{-t}(p)}}: N_{\phi^{-t}(p)} \rightarrow N_{p},
\end{aligned}
$$

and Lyapunov-type numbers, introduced in [3], are assigned to each point $p \in M$ as follows:

$$
\nu(p):=\limsup _{t \rightarrow \infty}\left\|B_{t}(p)\right\|^{1 / t}, \quad \sigma(p):=\limsup _{t \rightarrow \infty} \frac{\ln \left\|A_{t}(p)\right\|}{-\ln \left\|B_{t}(p)\right\|} .
$$

The number $\nu(p)$ measures the "exponential of the normal contraction rate" while $\sigma(p)$ compares the normal and tangential contraction rates. Both of these numbers are constant on orbits. Moreover, the Lyapunov-type numbers of an orbit are dominated by the supremum of the Lyapunov-type numbers on its $\alpha$-limit set. Thus, to prove that $M$ is normally hyperbolic, it suffices to compute the type numbers on the limit sets of the flow that are contained in $M$. A basic persistence result of Fenichel [3] states that if for all $p \in M$, we have $\nu(p)<1$ and $\sigma(p)<1 / k$ for some positive integer $k$, then the manifold $M$ persists under small $C^{1}$ perturbations by $C^{k}$ vector fields. Moreover, the perturbed manifold is $C^{k}$. Let us mention that $M$, with the hypotheses of Fenichel's theorem is called $k$-normally hyperbolic. We will also use an equivalent formulation of $k$-normal hyperbolicity introduced by Hirsch, Pugh, and Shub [7]. A specialization of their definition to our perturbation problem is given below in display (8.11).

The persistence results just mentioned are widely applicable. However, in the perturbation problem (1.1) mentioned in the introduction, the existence of an invariant manifold cannot be obtained by a direct application of these persistence results due to the singular nature of the perturbation terms. Also, in the setting of the auxiliary family (1.3), the persistence result does not guarantee the existence of an invariant manifold up to $\delta=\epsilon$. On the other hand, in combination with an appropriate continuation method, the full strength of the persistence theory can be exploited to study perturbation problems of this type.

The idea of the continuation method is simple. To describe it, let us consider the smooth family $E^{\epsilon}$ of differential equations

$$
\dot{x}=f(x, \epsilon) .
$$

Suppose that $E^{0}$ has a $k$-normally hyperbolic invariant manifold $M(0)$, and we wish to know if there is a corresponding family $M(\epsilon)$ of $k$-normally hyperbolic invariant manifolds that can be continued to some preassigned value of $\epsilon$, say, $\epsilon=1$. In this 
case, we can proceed in the following manner: Define $A$ to be the set of all $\epsilon$ in the unit interval such that, for all $\epsilon^{\prime} \in[0, \epsilon]$, the corresponding system $E^{\epsilon^{\prime}}$ has a $k$-normally hyperbolic invariant manifold $M\left(\epsilon^{\prime}\right)$, and then prove that $A$ is nonempty, open, and closed. Because $M(0)$ is a $k$-normally hyperbolic invariant manifold for $E^{0}, A$ is not empty. The fact that $A$ is open follows from the general persistence theory. Thus, all that remains is to show that $A$ is closed; that is, if $\epsilon_{*}$ is the supremum of $A$, then $\epsilon_{*} \in A$. This can be accomplished in two steps: Prove that the system $E^{\epsilon_{*}}$ has a $C^{1}$ invariant manifold; then, prove that this invariant manifold is $k$-normally hyperbolic. Since we have a family of $k$-normally hyperbolic invariant manifolds $M(\epsilon)$ defined for $\epsilon^{\prime} \in\left[0, \epsilon_{*}\right)$, the first step can be proved by showing that these manifolds are realized as graphs of an equicontinuous family of $C^{1}$ functions. The $C^{k}$ smoothness of the limit manifold is obtained as a consequence of the second step which can be proved by checking the definition of $k$-normal hyperbolicity.

Let us consider a general family of the form

$$
\dot{x}=f(x, \epsilon)+\epsilon g(x, \epsilon),
$$

where, for some $\delta_{0}>0$, the system $\dot{x}=f(x, \delta)$ has a normally hyperbolic invariant manifold for $0<\delta \leq \delta_{0}$. Kopell [8] studies a model equation that can be viewed as a special case of this family. To apply the general continuation method just described, she introduces an auxiliary family, which in our more general context would be

$$
\dot{x}=f(x, \delta)+\epsilon g(x, \epsilon) .
$$

For this auxiliary system, if $\delta \in\left(0, \delta_{0}\right)$, then there is some $\epsilon(\delta)>0$ such that, for $0 \leq \epsilon<\epsilon(\delta)$, the corresponding member of the auxiliary family has a normally hyperbolic invariant manifold $M(\delta, \epsilon)$. The idea is to fix some $\delta>0$ sufficiently small so that continuation of normally hyperbolic invariant manifolds relative to the parameter $\epsilon$ can be carried out all the way to $\epsilon=\delta$. If this continuation is possible, then the member of the original family corresponding to $\epsilon=\delta$ has an invariant manifold.

In [8] (see also Wiggins [12, pp. 168-170]), a continuation theorem is stated for a family of the type described above, but of a more special form. However, the strategy of the proof of this theorem contains a gap. To describe the gap it is not necessary to consider the precise form of the equations or the hypotheses of the theorem. Rather, we will explain the problem in a general framework. Indeed, let us consider the parameter space of a family of differential equations and the subspace $\mathcal{N}$ corresponding to family members with a normally hyperbolic invariant manifold. Suppose that a path in $\mathcal{N}$ approaches the boundary of $\mathcal{N}$. Also, consider the supremum of each of the Lyapunov-type numbers $\nu$ and $\sigma$ taken individually over the orbits of each normally hyperbolic invariant manifold in a continuous family. It is perhaps natural to suspect that the limit of at least one of these suprema converges to the number 1 as the path approaches the boundary. In other words, one might assume that the normal hyperbolicity is lost at the boundary only in this manner. However, this is not always the case. In fact, there may be paths for which the corresponding continuous family of normally hyperbolic invariant manifolds has both Lyapunov-type numbers uniformly bounded below one but the family of invariant manifolds does not converge to a $C^{1}$ manifold. Thus, in a continuation argument, it is required to prove that smooth invariant manifolds exist over the entire continuation interval and that all these manifolds are normally hyperbolic. The following example clearly shows why both requirements must be satisfied. 
Consider a planar system

$$
\dot{x}=f(x)
$$

with a homoclinic loop at a hyperbolic saddle $p$ whose eigenvalues $\alpha$ and $\beta$ are such that $\alpha+\beta<0$. In particular, the loop will be stable from the inside and the divergence of the vector field $f$ at $p$ is negative. Now add a one parameter family of perturbations $g(x, \epsilon)$ so that for $-1<\epsilon<0$ there is a limit cycle $\Gamma(\epsilon)$ that limits on the loop as $\epsilon$ approaches zero from the left and such that there is no limit cycle for $\epsilon>0$. If we view $\Gamma(\epsilon)$ as an invariant manifold, then the corresponding Lyapunov-type number $\sigma(\epsilon)$ is identically zero. Also, the Lyapunov-type number $\nu(\epsilon)$ of $\Gamma(\epsilon)$ is exactly its Floquet multiplier; that is,

$$
\nu(\epsilon)=e^{\frac{1}{T(\epsilon)} \int_{0}^{T(\epsilon)} \operatorname{div} f(\gamma(t, \epsilon)) d t}
$$

where $t \mapsto \gamma(t, \epsilon)$ is a periodic solution corresponding to the limit cycle and $T(\epsilon)$ is its period. Since the periodic solution spends most of its time near the hyperbolic saddle point, the Lyapunov-type number $\nu(\epsilon)$ approaches $e^{\alpha+\beta}$ as $\epsilon \rightarrow 0^{-}$. In particular, both Lyapunov-type numbers are bounded above by some number that is strictly less than one. But, the limit of the hyperbolic limit cycles is the nonsmooth homoclinic loop. Thus, in general, it is not enough to obtain uniform estimates on the Lyapunovtype numbers to ensure that a family of normally hyperbolic invariant manifolds can be continued.

4. Statement of main result. In this section we will state the continuation theorem that will be proved in this paper.

To obtain an invariant manifold for system (2.9) using a perturbation argument, it is useful to have an unperturbed system with an invariant manifold. As given, system (2.9), even after rescaling time, is degenerate in the limit as $\mu$ approaches zero. To remedy this problem, we will change coordinates and also rescale time so as to obtain a suitable perturbation problem.

Let us suppose that $\mu \neq 0$. Introduce new coordinates $\ell=\mu \hat{\rho}, \tau=\mu^{2} \varphi$, and a slow time $s=\mu^{2} t$, and note that system (2.9) is equivalent to the system

$$
\begin{aligned}
\hat{\rho}^{\prime} & =p(\sigma) \hat{\rho}+r(\sigma)+\mu^{2} q(\sigma) \hat{\rho}^{2}+\mu \widehat{R}\left(\mu \hat{\rho}, \sigma, \tau / \mu^{2}, \mu\right), \\
\sigma^{\prime} & =\lambda \hat{\rho}+g(\sigma)+\mu^{2} \nu \hat{\rho}^{2}+\mu \widehat{S}\left(\mu \hat{\rho}, \sigma, \tau / \mu^{2}, \mu\right), \\
\tau^{\prime} & =1
\end{aligned}
$$

where the symbol " " " denotes differentiation with respect to $s$. Let us also use the new coordinate $\rho:=\lambda \hat{\rho}+g(\sigma)$ to express system (4.1) in the form

$$
\begin{aligned}
& \rho^{\prime}=\Delta(\sigma) \rho+\Lambda(\sigma)+\mu R\left(\rho, \sigma, \tau / \mu^{2}, \mu\right) \\
& \sigma^{\prime}=\rho+\mu S\left(\rho, \sigma, \tau / \mu^{2}, \mu\right) \\
& \tau^{\prime}=1
\end{aligned}
$$

where

$$
\Lambda(\sigma):=\lambda r(\sigma)-p(\sigma) g(\sigma), \quad \Delta(\sigma):=p(\sigma)+g^{\prime}(\sigma),
$$

and the functions $R$ and $S$ are $2 \pi$ periodic in $\sigma$ and $2 \pi m \mu^{2} / \Omega$ periodic in $\tau$. 
Let us write $\sigma \in \mathbb{S}^{1}$ to indicate that $\sigma$ is an angular variable in the interval $0 \leq \sigma \leq 2 \pi$ with the end points identified. Also, we will use the following hypothesis.

HyPOTHESIS 1. For each $\sigma \in \mathbb{S}^{1}, \Lambda(\sigma) \neq 0$ and $\Delta(\sigma)<0$.

THEOREM 4.1. If $k \geq 2$ is an integer, Hypothesis 1 holds, and $|\mu|>0$ is suffciently small, then system (4.2) has a k-normally hyperbolic invariant torus.

We note that the $k$-normal hyperbolicity of the invariant torus in the conclusion of Theorem 4.1 implies that the invariant torus is $C^{k}$; see [7]. Also, as an immediate corollary of Theorem 4.1-just reverse the direction of time - the same conclusion holds under the assumption that, for each $\sigma \in \mathbb{S}^{1}, \Lambda(\sigma) \neq 0$ and $\Delta(\sigma)>0$. Also, if we assume that $g(\sigma) \equiv 0$, as in Theorem 2.1 and if we assume that $r$ has no zeros as in Hypothesis 1, then Theorem 4.1 is a generalization of Theorem 2.1. Indeed, the inequalities required in Theorem 2.1 are all replaced by the requirement that $p$ has no zeros.

Finally, we mention that Theorem 4.1 is not valid if Hypothesis 1 is modified to allow the function $\Lambda$ to have zeros. In fact, to obtain an analogue of Theorem 4.1 in case $\Lambda$ has zeros, additional restrictions must be imposed. The formulation of the "right" hypotheses needed to prove an analogue of Theorem 4.1 in this case remains an interesting open problem.

The main idea of our proof of Theorem 4.1 is to view system (4.2) as a perturbation of the system

$$
\begin{aligned}
\rho^{\prime} & =\Delta(\sigma) \rho+\Lambda(\sigma), \\
\sigma^{\prime} & =\rho, \\
\tau^{\prime} & =1
\end{aligned}
$$

and to show that the unperturbed system (4.4) has a normally hyperbolic invariant torus that continues to an invariant torus for system (4.2). We also note that the invariant torus for system (4.4) is the suspension of a normally hyperbolic invariant (simple closed) curve for the system

$$
\begin{aligned}
& \rho^{\prime}=\Delta(\sigma) \rho+\Lambda(\sigma), \\
& \sigma^{\prime}=\rho .
\end{aligned}
$$

5. Existence of an invariant curve. In this section we will prove that the unperturbed system (4.5) has a normally hyperbolic invariant curve. More precisely, we have the following theorem.

THEOREM 5.1. If Hypothesis 1 holds, then the system (4.5) has a $C^{\infty}$ normally hyperbolic invariant simple closed curve given as the graph of a $C^{\infty}$ function of the angular variable.

There are several ways to prove Theorem 5.1. For example, a positively invariant annulus can be constructed, and the existence of a limit cycle can be proved using the Poincaré-Bendixson theorem. While the proof given below is more involved, it serves to illustrate the continuation technique that will be used in our proof of the existence of an invariant torus for system (4.1).

Our idea is to find a family of systems that includes system (4.5), to find a member of the family that has a normally hyperbolic invariant manifold, and then to continue this manifold through the family to the system (4.5).

Let us consider the family

$$
\begin{aligned}
& \rho^{\prime}=\Delta(\sigma) \rho+\epsilon \Lambda(\sigma), \\
& \sigma^{\prime}=\rho .
\end{aligned}
$$


We will use the next obvious lemma.

Lemma 5.2. If Hypothesis 1 holds and $\epsilon>0$, then system (5.1) has no rest point.

Theorem 5.1 is an immediate consequence of the following lemma.

LEMMA 5.3. If Hypothesis 1 holds for system (4.5), then system (5.1) has a $C^{\infty}$ normally hyperbolic invariant curve that is given as the graph of a $C^{\infty}$ function of the angular variable for all $\epsilon \in[0,1]$.

Proof. By Hypothesis 1 , the function $\Lambda$ does not vanish. Without loss of generality, we will assume that $\Lambda(\sigma)>0$ for all $\sigma$. Also, by Hypothesis 1 , the curve given by $\{(\rho, \sigma): \rho=0\}$ is a normally hyperbolic invariant manifold for the member of the family (5.1) at $\epsilon=0$. Following the strategy discussed in section 3 let us consider the set $A$ of all $\epsilon$ in the closed unit interval such that for all $\epsilon^{\prime} \in[0, \epsilon]$ the corresponding member of the family (5.1) has a normally hyperbolic invariant closed curve $\gamma^{\epsilon}$ given as the graph of a $C^{\infty}$ function $h^{\epsilon}$ of the angular variable. We will show that $A$ is nonempty, open, and closed. This implies $A=[0,1]$.

Because the invariant curve given by $\{(\rho, \sigma): \rho=0\}$ is normally hyperbolic for the family member at $\epsilon=0$, we have that $0 \in A$ and therefore $A$ is not empty. The fact that $A$ is open follows from the persistence results for normally hyperbolic invariant manifolds. Let us define $\epsilon_{*}=\sup A$. To complete the proof we will show that $\epsilon_{*} \in A$; that is, $A$ is closed.

Consider the family of curves

$$
\Gamma(\kappa):=\left\{(\rho, \sigma) \in \mathbb{R}^{2}: \rho-\kappa \Lambda(\sigma)=0\right\},
$$

where $\kappa \in \mathbb{R}$. Note that the curve $\Gamma(\kappa)$ is the graph of a periodic function of the angular variable. Thus, it separates the phase cylinder given by $(\rho, \sigma) \in \mathbb{R} \times \mathbb{S}^{1}$. Moreover, on $\Gamma(\kappa)$, by a straightforward computation, it follows that the dot product of the gradient of the function $(\rho, \sigma) \mapsto \rho-\kappa \Lambda(\sigma)$ and the vector field corresponding to the differential equation $E^{\epsilon}$ is given by

$$
\left(-\kappa^{2} \Lambda^{\prime}(\sigma)+\kappa \Delta(\sigma)+\epsilon\right) \Lambda(\sigma) .
$$

For $\epsilon \in\left[\epsilon_{*} / 2, \epsilon_{*}\right)$, there exists $\kappa_{0}>0$ such that the coefficient of $\Lambda(\sigma)$ in (5.2) with $\kappa=\kappa_{0}$ is positive for all $\sigma$. Hence, the vector field corresponding to $E^{\epsilon}$ is transverse to the curve $\Gamma\left(\kappa_{0}\right)$. Because the function $\Lambda$ is positive, it follows that $\gamma^{\epsilon}$ lies above the curve $\Gamma\left(\kappa_{0}\right)$; that is, $h^{\epsilon}(\sigma)>\kappa_{0} \Lambda(\sigma)$. Similarly, if $\nu \in \mathbb{R}$ is sufficiently large, then $\gamma^{\epsilon}$ lies below the curve $\{(\rho, \sigma): \rho=\nu\}$. In particular, the set of functions $\mathcal{S}:=\left\{h^{\epsilon}: \epsilon \in\left[\epsilon_{*} / 2, \epsilon_{*}\right)\right\}$ is uniformly bounded.

Using the invariance, the function $h^{\epsilon}$ satisfies the differential equation

$$
h_{\sigma}^{\epsilon}(\sigma)=\Delta(\sigma)+\epsilon \frac{\Lambda(\sigma)}{h^{\epsilon}(\sigma)} .
$$

Thus, we have that $\left|h_{\sigma}^{\epsilon}\right| \leq|\Delta(\sigma)|+\epsilon_{*} / \kappa_{0}$ uniformly for $\epsilon \in\left[\epsilon_{*} / 2, \epsilon_{*}\right)$, and, as a result, the set $\mathcal{S}$ is equicontinuous in the $C^{0}$ norm. By Arzela's theorem, there is a subsequence that converges to a continuous function $h^{\epsilon_{*}}$.

We claim that the graph of $h^{\epsilon_{*}}$ is an invariant set for $E^{\epsilon_{*}}$. To prove the claim, let $s \mapsto\left(\rho^{\epsilon}(s, q), \sigma^{\epsilon}(s, q)\right)$ denote the solution of $E^{\epsilon}$ such that $\sigma^{\epsilon}(0, q)=q$ and $\rho^{\epsilon}(0, q)=h^{\epsilon}(q)$, and let us suppose that $h^{\epsilon_{n}}$ converges to $h^{\epsilon_{*}}$. If $s \in \mathbb{R}$, then, using the continuity of the flow with respect to parameters, we have that $\sigma^{\epsilon_{n}}(s, q) \rightarrow$ $\sigma^{\epsilon_{*}}(s, q)$ and $\rho^{\epsilon_{n}}(s, q) \rightarrow \rho^{\epsilon_{*}}(s, q)$. By passing to the limit as $n \rightarrow \infty$ in the identity 
$\rho^{\epsilon_{n}}(s, q)=h^{\epsilon_{n}}\left(\sigma^{\epsilon_{n}}(s, q)\right)$, we have $\rho^{\epsilon_{*}}(s, q)=h^{\epsilon_{*}}\left(\sigma^{\epsilon_{*}}(s, q)\right)$. Thus, it follows that the graph of $h^{\epsilon_{*}}$ is an invariant set for $E^{\epsilon_{*}}$. Because this invariant set is a single orbit of the differential equation, it is $C^{\infty}$. Moreover, because the function $\Delta$ is everywhere negative, this invariant set is normally hyperbolic - it is a hyperbolic limit cycle.

6. An a priori estimate for perturbed manifolds. The following proposition, which perhaps has independent interest, will play a key role in our proof of Theorem 4.1. While the statement of this proposition is natural, we do not know if it appears in the literature. Thus, we will give a complete proof in the appendix.

Proposition 6.1. Consider a smooth planar vector field

$$
x^{\prime}=f(x)
$$

with a periodic solution $t \mapsto x(t, p)$ of period $\omega$ corresponding to the periodic orbit $\Gamma$. If $\Gamma$ is hyperbolic and asymptotically stable, that is,

$$
b:=\int_{0}^{\omega} \operatorname{tr} D f(x(t, p)) d t<0,
$$

then there exist a neighborhood $N$ of $\Gamma$ and a constant $C>0$ such that for every smooth function $g: N \rightarrow \mathbb{R}^{2}$ for which the differential equation

$$
x^{\prime}=f(x)+g(x)
$$

has an invariant set $\bar{\Gamma} \subset N$, we have the following a priori estimate:

$$
\sup \{d(x, \Gamma): x \in \bar{\Gamma}\} \leq C\|g\|_{C^{0}},
$$

where $\|g\|_{C^{0}}$ is the supremum norm over $N$ and d denotes the usual distance between sets.

Proposition 6.2. Consider a planar differential equation

$$
x^{\prime}=f(x)
$$

with a hyperbolic limit cycle $\Gamma$ of period $T>0$, and let $\tau$ be an angular variable modulo $T$. If $\Gamma$ is asymptotically stable, then there is a neighborhood $N \subset \mathbb{R}^{2} \times \mathbb{R}$ of the corresponding invariant torus $M$ for the system

$$
x^{\prime}=f(x), \quad \tau^{\prime}=1
$$

and a constant $C>0$ such that for every smooth function $g: N \rightarrow \mathbb{R}^{2}$, with $g(x, \tau+$ $T)=g(x, \tau)$ for each $x \in \mathbb{R}^{2}$ and all $\tau \in \mathbb{R}$, and for which the system

$$
x^{\prime}=f(x)+g(x, \tau), \quad \tau^{\prime}=1
$$

has an invariant set $\bar{M} \subset N$, we have the a priori estimate

$$
\sup \{d(x, M): x \in \bar{M}\} \leq C\|g\|_{C^{0}} .
$$


7. Existence of invariant tori. In this section we will state our result on the existence of an invariant torus for the systems (2.9) and (4.2) as well as the main lemmas that we will use to prove it. In fact, we will prove the existence of invariant tori for systems of the more general form

$$
\begin{aligned}
\rho^{\prime} & =f(\rho, \sigma)+\mu R\left(\rho, \sigma, \tau / \mu^{2}, \mu\right), \\
\sigma^{\prime} & =g(\rho, \sigma)+\mu S\left(\rho, \sigma, \tau / \mu^{2}, \mu\right), \\
\tau^{\prime} & =1,
\end{aligned}
$$

where $|\mu|>0$, where $f, g, R$, and $S$ (redefined for this section) are all $C^{r}$ functions that are $2 \pi$-periodic functions of the angular variable $\sigma$, and where the functions $t \mapsto R(\rho, \sigma, t, \mu)$ and $t \mapsto S(\rho, \sigma, t, \mu)$ are $2 \pi m / \Omega$-periodic. For system (7.1), we view $\tau$ as an angular variable modulo $2 \pi m \mu^{2} / \Omega$ and we let $s$ denote the independent variable. Note that the slow time system (4.1) equivalent to system (2.9) is a special case of the differential (7.1). For a general discussion of integral manifolds for nonautonomous systems, see [1], [6].

To state our main result for system (7.1), let us consider the corresponding unperturbed system

$$
\rho^{\prime}=f(\rho, \sigma), \quad \sigma^{\prime}=g(\rho, \sigma),
$$

and the following hypothesis.

HYPOTHESIS 2. System (7.2) has an attracting hyperbolic limit cycle $\Gamma$ that is the graph of a function of the angular variable $\sigma$.

THEOREM 7.1. If $k$ is an integer such that $2 \leq k \leq r$ and the system (7.2) satisfies Hypothesis 2, then, for sufficiently small $|\mu|>0$, system (7.1) has a $k$ normally hyperbolic invariant manifold that is the graph of a function of the angular variables $\sigma$ and $\tau$.

The proof of Theorem 7.1 is given in the remaining sections of this paper using the lemmas that are stated below. Let us note at this point that it suffices to prove Theorem 7.1 for the case $\mu>0$. The result for $\mu<0$ follows from the first case by redefining the functions $R$ and $S$ in an obvious manner. Thus, we will consider only the case $\mu>0$.

Let us consider the auxiliary family $E^{\epsilon, \mu}$ given by

$$
\begin{aligned}
\rho^{\prime} & =f(\rho, \sigma)+\epsilon R\left(\rho, \sigma, \tau / \mu^{2}, \mu\right), \\
\sigma^{\prime} & =g(\rho, \sigma)+\epsilon S\left(\rho, \sigma, \tau / \mu^{2}, \mu\right), \\
\tau^{\prime} & =1 .
\end{aligned}
$$

Note that, by our assumption, the suspended system

$$
\begin{aligned}
\rho^{\prime} & =f(\rho, \sigma), \\
\sigma^{\prime} & =g(\rho, \sigma), \\
\tau^{\prime} & =1,
\end{aligned}
$$

where $\tau$ is viewed as a new angular variable modulo $2 \pi m \mu^{2} / \Omega$, has a normally hyperbolic torus that is a graph over the two angular variables $\sigma$ and $\tau$. For our analysis we will consider the torus as a submanifold of the phase cylinder $\mathcal{C}$ given by $(\rho, \sigma, \tau) \in \mathbb{R}^{3}$, where $\sigma$ and $\tau$ are viewed as the angular variables defined above. Topologically, $\mathcal{C}$ is the product of the real line with a two-dimensional torus. 
For each $\mu>0$, let us denote by $A^{\mu}$ the maximal interval with left endpoint at $\epsilon=0$ such that the system $E^{\epsilon, \mu}$ has a $k$-normally hyperbolic invariant manifold, $k \geq 2$, as defined in [7]; see also display (8.11), given as the graph of a $C^{k}$ function of the angular variables. Using the continuation strategy outlined in section 3 , let us note that, for each $\mu>0$, the set $A^{\mu}$ contains a nonempty relatively open interval with left endpoint $\epsilon=0$. Moreover, if $\epsilon \in A^{\mu}$, then, by the general persistence results for normally hyperbolic invariant manifolds, there is an open interval containing $\epsilon$ that is contained in $A^{\mu}$. Thus, Theorem 7.1 is an immediate consequence of the following proposition.

Proposition 7.2. Suppose that $\mu>0$ and $A^{\mu}$ is the maximal interval with left endpoint at $\epsilon=0$ such that the system $E^{\epsilon, \mu}$ has a $k$-normally hyperbolic invariant manifold, $k \geq 2$, that is the graph of a $C^{k}$ function of the angular variables. If $\mu>0$ is sufficiently small and if $\epsilon_{*} \leq \mu$ is the least upper bound of a relatively open interval with left endpoint $\epsilon=0$ in $A^{\mu}$, then $\epsilon_{*} \in A^{\mu}$.

Proposition 7.2 is a consequence of the following three lemmas.

Lemma 7.3. With the hypotheses and notation of Proposition 7.2, the system $E^{\epsilon_{*}, \mu}$ has an invariant manifold $M\left(\epsilon_{*}, \mu\right)$ given as the graph of a $C^{1}$ function of the angular variables.

Lemma 7.4. If $M\left(\epsilon_{*}, \mu\right)$ is the invariant manifold in Lemma 7.3, then it has an invariant normal bundle.

LEMma 7.5. If $M\left(\epsilon_{*}, \mu\right)$ is the invariant manifold in Lemma 7.3, then $M\left(\epsilon_{*}, \mu\right)$ is $k$-normally hyperbolic. In particular, $M\left(\epsilon_{*}, \mu\right)$ is $C^{k}$ and $\epsilon_{*} \in A^{\mu}$.

8. Notation and preliminary results. Lemmas (7.3)-(7.5) will be proved in the following sections. In this section we will define new notation and obtain some preliminary results that will be used in all three proofs. For the remainder of this section let us assume that $\mu>0$ and $\epsilon \geq 0$ are fixed, and that system (7.3) has a normally hyperbolic invariant torus $M:=M(\epsilon, \mu)$ given as the graph of the $C^{1}$ function $h^{\epsilon}$ of the angular variables.

8.1. Normal splitting and variational solutions. The general results for normally hyperbolic invariant manifolds give the existence of an invariant splitting of $T_{M} \mathcal{C}$, the tangent bundle of the phase cylinder $\mathcal{C}$ restricted to this normally hyperbolic invariant torus, as a direct sum of the tangent bundle of the invariant torus $M$ and an invariant normal bundle.

For notational convenience, let us define new functions

$$
\begin{aligned}
& F(\rho, \sigma, \tau, \mu, \epsilon):=f(\rho, \sigma)+\epsilon R\left(\rho, \sigma, \tau / \mu^{2}, \mu\right), \\
& G(\rho, \sigma, \tau, \mu, \epsilon):=g(\rho, \sigma)+\epsilon S\left(\rho, \sigma, \tau / \mu^{2}, \mu\right),
\end{aligned}
$$

and let us suppose that the invariant torus $M(\epsilon, \mu)$ is given as the graph of the function $(\sigma, \tau) \mapsto h^{\epsilon}(\sigma, \tau)$.

The vector field

$$
\mathcal{X}_{1}^{\epsilon}(\sigma, \tau):=\left(\begin{array}{c}
F\left(h^{\epsilon}(\sigma, \tau), \sigma, \tau, \mu, \epsilon\right) \\
G\left(h^{\epsilon}(\sigma, \tau), \sigma, \tau, \mu, \epsilon\right) \\
1
\end{array}\right)
$$

is clearly tangent to $M(\epsilon, \mu)$. Also, as is easily seen by computing the tangents to 
each curve on $M(\epsilon, \mu)$ given by $\sigma \mapsto\left(h^{\epsilon}(\sigma, \tau), \sigma, \tau\right)$ for some fixed $\tau$, the vector field

$$
\mathcal{X}_{2}^{\epsilon}(\sigma, \tau):=\left(\begin{array}{c}
h_{\sigma}^{\epsilon}(\sigma, \tau) \\
1 \\
0
\end{array}\right)
$$

is tangent to $M(\epsilon, \mu)$. Moreover, if $\xi=\left(h^{\epsilon}(\sigma, \tau), \sigma, \tau\right)$, then $\mathcal{X}_{1}^{\epsilon}(\sigma, \tau)$ and $\mathcal{X}_{2}^{\epsilon}(\sigma, \tau)$ span the corresponding fiber $T_{\xi} M(\epsilon, \mu)$ of the tangent bundle of $M(\epsilon, \mu)$.

To determine the contraction rates for the flow on the invariant torus $M(\epsilon, \mu)$, we must consider the solutions of the first variational equation for the system (7.3). If

$$
s \mapsto \gamma^{\epsilon}(s, q):=\left(h^{\epsilon}\left(\sigma^{\epsilon}(s, q), \tau(s)\right), \sigma^{\epsilon}(s, q), \tau(s)\right)
$$

is the solution of the system $(7.3)$ with $\gamma^{\epsilon}(0, q)=\left(h^{\epsilon}(q, 0), q, 0\right)$, then the variational equation along the solution $s \mapsto \gamma^{\epsilon}(s, q)$ is given by

$$
\left(\begin{array}{c}
u^{\prime} \\
v^{\prime} \\
w^{\prime}
\end{array}\right)\left(\begin{array}{ccc}
F_{\rho} & F_{\sigma} & F_{\tau} \\
G_{\rho} & G_{\sigma} & G_{\tau} \\
0 & 0 & 0
\end{array}\right)\left(\begin{array}{c}
u \\
v \\
w
\end{array}\right),
$$

where the argument of each function in the system matrix is given by

$$
\left(h^{\epsilon}\left(\sigma^{\epsilon}(s, q), \tau(s)\right), \sigma^{\epsilon}(s, q), \tau(s), \mu, \epsilon\right) .
$$

Proposition 8.1. The variational (8.5) along the solution (8.4) on the invariant torus $M(\epsilon, \mu)$ has two independent solutions given by

$$
X_{1}(s):=\mathcal{X}_{1}^{\epsilon}\left(\gamma^{\epsilon}(s, q)\right), \quad X_{2}(s):=y^{\epsilon}(s, q) \mathcal{X}_{2}^{\epsilon}\left(\gamma^{\epsilon}(s, q)\right),
$$

where

$$
y^{\epsilon}(t, q):=\exp \left(\int_{0}^{t}\left(G_{\rho} h_{\sigma}^{\epsilon}+G_{\sigma}\right) d s\right)
$$

and the argument of $F$ and $G$ is given in display (8.6). Moreover, $X_{1}(s)$ and $X_{2}(s)$ span the tangent space of the invariant torus at each point along the solution (8.4).

Proof. The solution $X_{1}(s)$ is just the evaluation of the vector field corresponding to the base differential (7.3) along one of its integral curves. Thus, as is well known, it is a solution of the variational equation.

To obtain the second solution, let us recall that the invariant torus is given as a graph over the angular variables. In particular, the differential equation expressed in the corresponding local coordinates - the projection $(\rho, \sigma, \tau) \mapsto(\sigma, \tau)$ restricted to the graph is the coordinate map - is given by

$$
\sigma^{\prime}=G\left(h^{\epsilon}(\sigma, \tau), \sigma, \tau, \mu, \epsilon\right), \quad \tau^{\prime}=1 .
$$

The corresponding variational equation has the form

$$
v^{\prime}=\left(G_{\rho} h_{\sigma}^{\epsilon}+G_{\sigma}\right) v+\left(G_{\rho} h_{\tau}^{\epsilon}+G_{\tau}\right) w, \quad w^{\prime}=0 .
$$

One of its solutions is given by

$$
s \mapsto(v(s), w(s))=\left(y^{\epsilon}(s, q), 0\right) .
$$


As $\rho=h^{\epsilon}(\sigma, \tau)$ on the invariant torus, the corresponding solution of the variational equation in the original coordinates is given by $y^{\epsilon}(s, q) \mathcal{X}_{2}^{\epsilon}\left(\gamma^{\epsilon}(s, q)\right)$-substitute the general base solution into the local coordinate representation and then differentiate with respect to the initial condition.

In view of the fact that $\mathcal{X}_{1}^{\epsilon}$ and $\mathcal{X}_{2}^{\epsilon}$ are independent at each point of the manifold, and by virtue of the fact that $y^{\epsilon}$ is a positive function, the two solutions $X_{1}(s)$ and $X_{2}(s)$ are independent at each point along the solution $\gamma^{\epsilon}$.

Let $\Phi^{\epsilon}(s)$ denote the principal fundamental matrix solution of the variational equation (8.5) at $s=0$. By the general theory of normally hyperbolic invariant manifolds, there is a normal bundle over the invariant torus $M$ that is invariant under $\Phi^{\epsilon}(s)$. Because, $M$ has codimension one, the fiber dimension of the normal bundle is one. Also, let us consider the family of cylinders given by

$$
\mathcal{L}^{s}:=\{(\rho, \sigma, \tau): \tau=s\}
$$

and note that $\mathcal{L}:=\cup\left\{\mathcal{L}^{s}: s \in \mathbb{R}\right\}$ is a foliation of the phase cylinder that is invariant under the flow of system (7.3). Thus, it follows that $\mathcal{L}$ is also invariant for the variational equation, or equivalently, it is invariant under $\Phi^{\epsilon}(s)$. Because of the invariance of this foliation and the normal hyperbolicity, the fiber of the invariant normal bundle must be tangent to the leaf of this foliation that passes through the base point of the fiber. Also, the normal bundle of the embedded torus is trivial. Thus, it has a continuous nonzero section $\mathcal{X}_{0}^{\epsilon}$. Let us define

$$
X_{0}(s):=\mathcal{X}_{0}^{\epsilon}\left(\gamma^{\epsilon}(s, q)\right),
$$

where $\gamma^{\epsilon}$ is the solution defined in display (8.4). We remark here that the invariant normal bundle is required only to be continuous. In fact, in general it is not smooth.

To determine the growth rates required for the normal hyperbolicity, let us define

$$
\lambda_{1}(s):=\frac{\left|X_{1}(s)\right|}{\left|X_{1}(0)\right|}, \quad \lambda_{2}(s):=\frac{\left|X_{2}(s)\right|}{\left|X_{2}(0)\right|}, \quad \lambda_{3}(s):=\frac{\left|\Phi^{\epsilon}(s) X_{0}(0)\right|}{\left|X_{0}(0)\right|} .
$$

If $k$ is a positive integer, then the invariant torus $M(\epsilon, \mu)$ is $k$-normally hyperbolic, as defined in [7], provided that there are numbers $\beta>0$ and $c>0$ independent of the choice of the solution on $M(\epsilon, \mu)$ such that the following conditions are satisfied for $s \geq 0$ :

$$
\lambda_{3}(s) \leq c e^{-\beta s}, \quad \frac{\lambda_{3}(s)}{\lambda_{1}^{k}(s)} \leq c e^{-\beta s}, \quad \frac{\lambda_{3}(s)}{\lambda_{2}^{k}(s)} \leq c e^{-\beta s} .
$$

8.2. A formula for $\boldsymbol{\lambda}_{\mathbf{3}}(s)$. The vector function $s \mapsto X_{2}(s)$ defined in display (8.7) is a solution of the system (8.5). Define

$$
X_{2}^{\perp}(s):=\left(\begin{array}{ccc}
0 & -1 & 0 \\
1 & 0 & 0 \\
0 & 0 & 1
\end{array}\right) X_{2}(s),
$$

and note that there are smooth functions $s \mapsto a(s)$ and $s \mapsto b(s)$ such that

$$
\Phi^{\epsilon}(s) X_{2}^{\perp}(0)=a(s) X_{2}(s)+b(s) X_{2}^{\perp}(s) .
$$


Moreover, it is not difficult to compute the following formulas:

$$
\begin{aligned}
b(s) & =\frac{\left|X_{2}(0)\right|^{2}}{\left|X_{2}(s)\right|^{2}} \exp \left(\int_{0}^{s} \operatorname{tr} B(t) d t\right), \\
a^{\prime}(s) & =\frac{b(s)}{\left|X_{2}(s)\right|^{2}}\left(\left\langle B(s) X_{2}(s), X_{2}^{\perp}(s)\right\rangle+\left\langle B(s) X_{2}^{\perp}(s), X_{2}(s)\right\rangle\right), \\
a(0) & =0,
\end{aligned}
$$

where $B(s)$ is the system matrix of the linear system (8.5).

By the above remarks, the vector $X_{0}(s)$ is in the span of the linearly independent vectors $X_{2}(s)$ and $X_{2}^{\perp}(s)$. Thus, by an appropriate choice of the nonzero normal bundle section $\mathcal{X}_{0}^{\epsilon}$, there is a smooth function $s \mapsto \alpha(s)$ such that

$$
X_{0}(s)=\alpha(s) X_{2}(s)+X_{2}^{\perp}(s)
$$

and a smooth function $s \mapsto \lambda(s)$ such that

$$
\Phi^{\epsilon}(s) X_{0}(0)=\lambda(s) X_{0}(s) .
$$

By substitution of the identity (8.13) into (8.14) and by using the independence of $X_{2}$ and $X_{2}^{\perp}$, it follows that $\lambda(s) \alpha(s)=\alpha(0)+a(s)$ and $\lambda(s)=b(s)$. Hence, using the definition given in display (8.10), and the formulas obtained in this section, we have the following equalities:

$$
\begin{aligned}
\lambda_{3}(s) & =\lambda(s) \frac{\left|X_{0}(s)\right|}{\left|X_{0}(0)\right|}=\left(\frac{\left|X_{2}(0)\right|^{2}}{\left|X_{2}(s)\right|^{2}} \exp \left(\int_{0}^{s} \operatorname{tr} B(t) d t\right)\right) \frac{\left(\alpha^{2}(s)+1\right)^{1 / 2}\left|X_{2}(s)\right|}{\left(\alpha^{2}(0)+1\right)^{1 / 2}\left|X_{2}(0)\right|} \\
& =\frac{\left|X_{2}(0)\right|}{\left|X_{2}(s)\right|}\left(\frac{\alpha^{2}(s)+1}{\alpha^{2}(0)+1}\right)^{1 / 2} \exp \left(\int_{0}^{s} \operatorname{tr} B(t) d t\right) .
\end{aligned}
$$

8.3. Derivative estimates. Under the assumptions that $\mu>0$ and the unperturbed system (7.2) have a normally hyperbolic invariant manifold given as a graph of a function of the angular variables, we know that $E^{\epsilon, \mu}$, for sufficiently small $\epsilon>0$, has a normally hyperbolic invariant manifold given as the graph of a function $h^{\epsilon}$ of the angular variables. In this section, we will determine some a priori estimates on the size of the derivatives of $h^{\epsilon}$. We will first state and prove two lemmas. The first reduces the main estimate from the vector to the scalar case, while the second lemma gives certain properties of an operator equation for one of the derivatives that must be estimated.

8.3.1. A reduction lemma. The next lemma shows that it suffices to estimate the partial derivative $h_{\sigma}$ on a Poincaré section.

Lemma 8.2. Suppose that $\mu>0$ and $\epsilon_{*}>0$ and that $E^{\epsilon, \mu}$ has an invariant manifold given as the graph of the function $h^{\epsilon}$ of the angular variables for $0 \leq \epsilon<$ $\epsilon_{*} \leq \mu$. If there is a constant $C_{1}>0$ such that, for all angles $\sigma$ and $\tau$, the following estimates hold:

$$
\left|h^{\epsilon}(\sigma, \tau)-h^{0}(\sigma, \tau)\right|<C_{1} \epsilon, \quad\left|h_{\sigma}^{\epsilon}(\sigma, 0)-h_{\sigma}^{0}(\sigma, 0)\right|<C_{1} \epsilon,
$$

then for $\mu$ sufficiently small there is a constant $C_{2}>0$ such that

$$
\left|h_{\sigma}^{\epsilon}(\sigma, \tau)-h_{\sigma}^{0}(\sigma, \tau)\right|<C_{2} \epsilon
$$


Proof. Let us suppose first that $\dot{x}=F(x, \epsilon)$ is a smooth family of differential equations. If $x^{\epsilon}$ and $x^{0}$ are solutions of the members of this family corresponding to their superscripts, then by an application of Gronwall's inequality there is a constant $K>0$ such that

$$
\left|x^{\epsilon}(t)-x^{0}(t)\right|<K e^{K|t|}\left(\left|x^{\epsilon}(0)-x^{0}(0)\right|+\epsilon|t|\right) .
$$

Let $s \mapsto \phi(s,(\rho, \sigma, \tau), \epsilon)$ be the solution of the system $E^{\epsilon, \mu}$ with the initial condition $\phi(0,(\rho, \sigma, \tau), \epsilon)=(\rho, \sigma, \tau)$, and note that the solution $\gamma^{\epsilon}$ defined in display (8.4) is given by $\gamma^{\epsilon}(s, q)=\phi\left(s,\left(h^{\epsilon}(q, 0), q, 0\right), \epsilon\right)$. For each pair of angles $p$ and $\tau$ with $0 \leq \tau<2 \pi m \mu^{2} / \Omega$, there is a unique angle $q^{\epsilon}$ defined by the equation

$$
\left(h^{\epsilon}\left(q^{\epsilon}, 0\right), q^{\epsilon}, 0\right)=\phi\left(-\tau,\left(h^{\epsilon}(p, \tau), p, \tau\right), \epsilon\right) .
$$

By an application of the inequality (8.16) to the family of solutions (8.17), if $\mu$ is sufficiently small, then there is a constant $K_{1}>0$, that does not depend on the choice of $\tau$, such that

$$
\begin{aligned}
\left|h^{\epsilon}\left(q^{\epsilon}, 0\right)-h^{0}\left(q^{0}, 0\right)\right|+\left|q^{\epsilon}-q^{0}\right| & \leq K_{1}\left(\left|h^{\epsilon}(p, \tau)-h^{0}(p, \tau)\right|+\epsilon\right) \\
& \leq K_{1}\left(C_{1}+1\right) \epsilon
\end{aligned}
$$

In particular, there is a constant $K_{2}>0$ such that

$$
\left|q^{\epsilon}-q^{0}\right| \leq K_{2} \epsilon
$$

By inverting the flow in (8.17), we have that $\gamma^{\epsilon}\left(\tau, q^{\epsilon}\right)=\left(h^{\epsilon}(p, \tau), p, \tau\right)$. Using an obvious modification of the notation as well as the result of Proposition 8.1, let us consider the first variational equation for $E^{\epsilon, \mu}$ along the solution $s \mapsto \gamma^{\epsilon}\left(s, q^{\epsilon}\right)$ and the solution of this variational equation that is given by

$$
X_{2}^{\epsilon}\left(s, q^{\epsilon}\right)=y^{\epsilon}\left(s, q^{\epsilon}\right)\left(\begin{array}{c}
h_{\sigma}^{\epsilon}\left(\sigma^{\epsilon}\left(s, q^{\epsilon}\right), \tau(s)\right) \\
1 \\
0
\end{array}\right) .
$$

Note that its initial condition and its value at $s=\tau$ are given by

$$
X_{2}^{\epsilon}\left(0, q^{\epsilon}\right)=\left(\begin{array}{c}
h_{\sigma}^{\epsilon}\left(q^{\epsilon}, 0\right) \\
1 \\
0
\end{array}\right), \quad X_{2}^{\epsilon}\left(\tau, q^{\epsilon}\right)=y^{\epsilon}\left(\tau, q^{\epsilon}\right)\left(\begin{array}{c}
h_{\sigma}^{\epsilon}(p, \tau) \\
1 \\
0
\end{array}\right) .
$$

By an application of the inequality (8.16) to this family of solutions of the variational equation, if $\mu$ is sufficiently small, then there is a constant $K_{2}>0$ such that

$$
\begin{aligned}
\mid y^{\epsilon}\left(\tau, q^{\epsilon}\right) h_{\sigma}^{\epsilon}(p, \tau) & -y^{0}\left(\tau, q^{0}\right) h_{\sigma}^{0}(p, \tau)|+| y^{\epsilon}\left(\tau, q^{\epsilon}\right)-y^{0}\left(\tau, q^{0}\right) \mid \\
& \leq K_{2}\left(\left|h_{\sigma}^{\epsilon}\left(q^{\epsilon}, 0\right)-h_{\sigma}^{0}\left(q^{0}, 0\right)\right|+\epsilon\right) \\
& \leq K_{2}\left(\left|h_{\sigma}^{\epsilon}\left(q^{\epsilon}, 0\right)-h_{\sigma}^{0}\left(q^{\epsilon}, 0\right)\right|+\left|h_{\sigma}^{0}\left(q^{\epsilon}, 0\right)-h_{\sigma}^{0}\left(q^{0}, 0\right)\right|+\epsilon\right) .
\end{aligned}
$$

Moreover, by the hypothesis of the lemma, by inequality (8.19), and by the fact that $h_{\sigma}^{0}$ is Lipschitz, we have that there is a constant $K_{3}>0$ such that

$$
\left|y^{\epsilon}\left(\tau, q^{\epsilon}\right) h_{\sigma}^{\epsilon}(p, \tau)-y^{0}\left(\tau, q^{0}\right) h_{\sigma}^{0}(p, \tau)\right|+\left|y^{\epsilon}\left(\tau, q^{\epsilon}\right)-y^{0}\left(\tau, q^{0}\right)\right| \leq K_{3} \epsilon
$$


In particular, both summands on the left-hand side of the last inequality are bounded above by $K_{3} \epsilon$.

By a reverse triangle law estimate starting with the fact that the quantity

$$
\left|\left(y^{\epsilon}\left(\tau, q^{\epsilon}\right) h_{\sigma}^{\epsilon}(p, \tau)-y^{\epsilon}\left(\tau, q^{\epsilon}\right) h_{\sigma}^{0}(p, \tau)\right)-\left(y^{0}\left(\tau, q^{0}\right) h_{\sigma}^{0}(p, \tau)-y^{\epsilon}\left(\tau, q^{\epsilon}\right) h_{\sigma}^{0}(p, \tau)\right)\right|
$$

is bounded above by $K_{3} \epsilon$, by the inequality (8.20), and the fact that $h_{\sigma}^{0}$ is uniformly bounded, we find that there is a constant $K_{4}>0$ such that

$$
\left|y^{\epsilon}\left(\tau, q^{\epsilon}\right)\right|\left|h_{\sigma}^{\epsilon}(p, \tau)-h_{\sigma}^{0}(p, \tau)\right| \leq K_{3} \epsilon+\left|h_{\sigma}^{0}(p, \tau)\right|\left|y^{\epsilon}\left(\tau, q^{\epsilon}\right)-y^{0}\left(\tau, q^{0}\right)\right| \leq K_{4} \epsilon .
$$

By a second reverse triangle law estimate, we have that

$$
\left|y^{\epsilon}\left(\tau, q^{\epsilon}\right)\right|=\left|y^{0}\left(\tau, q^{0}\right)+\left(y^{\epsilon}\left(\tau, q^{\epsilon}\right)-y^{0}\left(\tau, q^{0}\right)\right)\right| \geq\left|y^{0}\left(\tau, q^{0}\right)\right|-K_{3} \mu .
$$

Also, if we take $\mu>0$ sufficiently small, then there is a constant $K_{5}>0$ such that $\left|y^{\epsilon}\left(\tau, q^{\epsilon}\right)\right|>K_{5}$. The result follows from this fact and the inequality (8.21).

8.3.2. The estimates. Some of the most important estimates that are required for the proof of our main result are given in the next lemma.

Lemma 8.3. Suppose Hypothesis 2 holds for the unperturbed system (7.2). There is a number $\mu>0$ and a constant $C>0$ such that if $\epsilon_{*}$ is as in Proposition 7.2, then

$$
\left|h^{\epsilon}-h^{0}\right|_{C^{0}}<C \epsilon, \quad\left|h_{\sigma}^{\epsilon}-h_{\sigma}^{0}\right|_{C^{0}} \leq C \epsilon, \quad\left|h_{\tau}^{\epsilon}\right|_{C^{0}} \leq C \epsilon
$$

for $0 \leq \epsilon<\epsilon_{*}$.

Proof. We will show that, if $\mu>0$ is sufficiently small, then there is a constant $C>0$ such that $\left|h_{\sigma}^{\epsilon}-h_{\sigma}^{0}\right|_{C^{0}} \leq C \epsilon$. By Lemma 8.2, it suffices to find a constant $C>0$ such that, for all $q \in \mathbb{S}^{1}$,

$$
\left|h_{\sigma}^{\epsilon}(q, 0)-h_{\sigma}^{0}(q, 0)\right| \leq C \epsilon .
$$

To prove this inequality, let us recall Proposition 8.1, and note that the function given by $s \mapsto\left(h_{\sigma}^{\epsilon}\left(\sigma^{\epsilon}(s, q), \tau(s)\right) y^{\epsilon}(s, q), y^{\epsilon}(s, q)\right)$ is a solution of the "subsystem" of system (8.5) given by

$$
\begin{aligned}
& u^{\prime}=\left(f_{\rho}+\epsilon R_{\rho}\right) u+\left(f_{\sigma}+\epsilon R_{\sigma}\right) v, \\
& v^{\prime}=\left(g_{\rho}+\epsilon S_{\rho}\right) u+\left(g_{\sigma}+\epsilon S_{\sigma}\right) v .
\end{aligned}
$$

If $\Psi^{\epsilon}(s, q):=\left(\psi_{i j}^{\epsilon}(s, q)\right)_{2 \times 2}$ is the principal fundamental matrix solution of (8.22) at $s=0$, then

$$
\left(\begin{array}{c}
h_{\sigma}^{\epsilon}\left(\sigma^{\epsilon}(s, q), \tau(s)\right) y^{\epsilon}(s, q) \\
y^{\epsilon}(s, q)
\end{array}\right)=\Psi^{\epsilon}(s, q)\left(\begin{array}{c}
h_{\sigma}^{\epsilon}(q, 0) \\
1
\end{array}\right)
$$

Therefore, we have

$$
h_{\sigma}^{\epsilon}\left(\sigma^{\epsilon}(s, q), \tau(s)\right)=\frac{\psi_{11}^{\epsilon}(s, q) h_{\sigma}^{\epsilon}(q, 0)+\psi_{12}^{\epsilon}(s, q)}{\psi_{21}^{\epsilon}(s, q) h_{\sigma}^{\epsilon}(q, 0)+\psi_{22}^{\epsilon}(s, q)} .
$$

Because the second angular argument $\tau$ will often be set to $\tau=0$, in the remainder of the proof we will suppress the second argument in expressions involving the functions $h^{\epsilon}$ and their partial derivatives whenever $\epsilon>0$ and the second angular argument is set to $\tau=0$. In addition, the function $h^{0}$ is constant with respect to $\tau$; 
thus we will always suppress its second angular argument and write $h^{0}$ as a function of the first angular variable only.

If $s \mapsto(\rho(s), \sigma(s))$ is a solution of the unperturbed system

$$
\rho^{\prime}=f(\rho, \sigma), \quad \sigma^{\prime}=g(\rho, \sigma)
$$

then the vector function

$$
s \mapsto\left(f\left(h^{0}(\sigma), \sigma\right), g\left(h^{0}(\sigma), \sigma\right)\right)
$$

is a solution of the corresponding variational equation (8.22) with $\epsilon=0$. Using the fact that $\rho(s)=h^{0}(\sigma(s))$ on the invariant manifold, and differentiating with respect to $s$, we find that

$$
f\left(h^{0}(\sigma(s)), \sigma(s)\right)=h_{\sigma}^{0}(\sigma(s)) g\left(h^{0}(\sigma(s)), \sigma(s)\right) .
$$

Thus, the function $s \mapsto\left(h_{\sigma}^{0}(\sigma(s)) g\left(h^{0}(\sigma(s)), \sigma(s)\right), g\left(h^{0}(\sigma(s)), \sigma(s)\right)\right)$ is a solution of the variational equation. Using the fundamental matrix solution $\Psi^{\epsilon}$ defined after display (8.22), we find that

$$
\psi_{21}^{0}(s, q) h_{\sigma}^{0}(q)+\psi_{22}^{0}(s, q)=\frac{g\left(h^{0}(\sigma(s, q)), \sigma(s, q)\right)}{g\left(h^{0}(q), \sigma(q)\right)} .
$$

In view of the hypothesis that $\sigma^{\prime}$ does not vanish, there is a number $M_{0}>0$ such that $\left|\psi_{21}^{0}(s, q) h_{\sigma}^{0}(q)+\psi_{22}^{0}(s, q)\right| \geq M_{0}$ for every $s \in \mathbb{R}$ and $q \in \mathbb{S}^{1}$.

By hypothesis, the unperturbed normally hyperbolic invariant manifold for $E^{0, \mu}$ is the suspension of an attracting hyperbolic limit cycle. In particular, the characteristic multiplier of the limit cycle is negative. Using the fact that the determinant of the fundamental matrix solution of the variational equation is proportional to the exponential of the integral of the divergence of the vector field evaluated along the limit cycle, it follows that there is some $T_{0}>0$ such that, for $t \geq T_{0}$, we have the inequality $\operatorname{det} \Psi^{0}(t, q) \leq(1 / 4) M_{0}^{2}$. If, in addition, $0<\mu^{2}<\Omega /(2 \pi m)$, then there is a positive integer $n$ such that $T_{0} \leq 2 \pi m n \mu^{2} / \Omega<T_{0}+1$. For definiteness, let $n=n(\mu)$ denote the smallest such integer, and define

$$
T:=T(\mu)=2 \pi m n \mu^{2} / \Omega .
$$

While $T$ will vary as $\mu>0$ is made sufficiently small so that new requirements are satisfied, the final value of $T$ is an integer multiple of the period of the perturbation terms in the corresponding differential equation $E^{\epsilon, \mu}$, the value of $T$ is bounded above and below, and $T$ approaches $T_{0}$ as $\mu$ decreases toward zero.

If we set $s=T$ in (8.23), then

$$
h_{\sigma}^{\epsilon}(p)=\frac{\psi_{11}^{\epsilon}\left(T, q^{\epsilon}\right) h_{\sigma}^{\epsilon}\left(q^{\epsilon}\right)+\psi_{12}^{\epsilon}\left(T, q^{\epsilon}\right)}{\psi_{21}^{\epsilon}\left(T, q^{\epsilon}\right) h_{\sigma}^{\epsilon}\left(q^{\epsilon}\right)+\psi_{22}^{\epsilon}\left(T, q^{\epsilon}\right)},
$$

where $q^{\epsilon}$ is defined by the relation

$$
p=\sigma^{\epsilon}\left(T, q^{\epsilon}\right) .
$$

Choose a bounded neighborhood $N$ of the graph of $h^{0}$ and the corresponding constant $C_{0}>0$ as in Proposition 6.2. Also, choose $r_{0}>0$ so small that if $\left|h^{\epsilon}-h^{0}\right|_{C^{0}}<$ $r_{0}$, then the graph of $h^{\epsilon}$ is in $N$, and note that

$$
K:=\sup \left\{\left|R\left(\rho, \sigma, \tau / \mu^{2}, \mu\right)\right|+\left|S\left(\rho, \sigma, \tau / \mu^{2}, \mu\right)\right|:(\rho, \sigma, \tau) \in N, 0<\mu^{2}<\frac{\Omega}{2 \pi m}\right\}
$$


is finite - the functions $R$ and $S$ are the perturbation terms of the system $E^{\epsilon, \mu}$ in display (7.3).

Choose $r>0$ sufficiently small so that, for $T_{0} \leq T<T_{0}+1$,

$$
\left|d_{21} \xi+d_{22}\right|>\frac{2}{3} M_{0}, \quad\left|\operatorname{det} D-\operatorname{det} \Psi^{0}(T, q)\right|<\frac{1}{8} M_{0}^{2}
$$

whenever $\xi$, a real number, $D$, a $2 \times 2$ real matrix, and $q \in \mathbb{S}^{1}$ are such that

$$
\left|\xi-h_{\sigma}^{0}(q)\right|<r, \quad\left|D-\Psi^{0}(T, q)\right|<r .
$$

The existence of $r$ with the required properties follows from the continuity of the map $(u, v, w) \mapsto|u v+w|$, the continuity of the determinant function, and the compactness of $\mathbb{S}^{1}$.

If we choose $\mu>0$ so small that $0<\mu^{2}<\Omega / 2 \pi m$ and $C_{0} K \mu<r_{0}$, then, by Proposition 6.2,

$$
\left|h^{\epsilon}-h^{0}\right|_{C^{0}}<C_{0} K \epsilon
$$

as long as $\left|h^{\epsilon}-h^{0}\right|_{C^{0}}<r_{0}$. Thus, we have that the inequality (8.28) holds for $0 \leq \epsilon<\epsilon_{*}$. Also, by an application of the Gronwall estimate (8.16) applied to the solutions $\gamma^{\epsilon}(-s, p)$ and $\gamma^{0}(-s, p)$ defined in display (8.4), we find that there is a constant $C_{1}>0$ such that

$$
\left|h^{\epsilon}\left(q^{\epsilon}\right)-h^{0}\left(q^{0}\right)\right|+\left|q^{\epsilon}-q^{0}\right| \leq C_{1}\left(\left|h^{\epsilon}(p)-h^{0}(p)\right|+\epsilon\right) .
$$

In view of the estimate in display (8.28), we conclude that there is a constant $C_{2}>0$ such that $\left|q^{\epsilon}-q^{0}\right| \leq C_{2} \epsilon$. By an application of the estimate (8.16) to the solutions $s \mapsto \Psi^{\epsilon}\left(s, q^{\epsilon}\right)$ and $s \mapsto \Psi^{0}\left(s, q^{0}\right)$ of the variational equation, we have, for some $C_{3}>0$, the inequality

$$
\left|\Psi^{\epsilon}\left(T, q^{\epsilon}\right)-\Psi^{0}\left(T, q^{0}\right)\right| \leq C_{3}\left(\left|q^{\epsilon}-q\right|+\epsilon\right)
$$

To obtain the estimates in the statement of the lemma, we will first prove the following claim.

Claim. There exists a constant $C>0$ such that, for $0 \leq \epsilon<\epsilon_{*}$, if $\left|h_{\sigma}^{\epsilon}-h_{\sigma}^{0}\right|_{C^{0}} \leq r$, then $\left|h_{\sigma}^{\epsilon}-h_{\sigma}^{0}\right|_{C^{0}} \leq C \epsilon$.

Proof of claim. Fix $p \in \mathbb{S}^{1}$, and let $q^{\epsilon}$ be as in (8.26). Using this notation and the identity (8.25), we have

$$
\begin{aligned}
\left|h_{\sigma}^{\epsilon}(p)-h_{\sigma}^{0}(p)\right| \leq & \left|\frac{\psi_{11}^{\epsilon}\left(T, q^{\epsilon}\right) h_{\sigma}^{\epsilon}\left(q^{\epsilon}\right)+\psi_{12}^{\epsilon}\left(T, q^{\epsilon}\right)}{\psi_{21}^{\epsilon}\left(T, q^{\epsilon}\right) h_{\sigma}^{\epsilon}\left(q^{\epsilon}\right)+\psi_{22}^{\epsilon}\left(T, q^{\epsilon}\right)}-\frac{\psi_{11}^{\epsilon}\left(T, q^{\epsilon}\right) h_{\sigma}^{0}\left(q^{\epsilon}\right)+\psi_{12}^{\epsilon}\left(T, q^{\epsilon}\right)}{\psi_{21}^{\epsilon}\left(T, q^{\epsilon}\right) h_{\sigma}^{0}\left(q^{\epsilon}\right)+\psi_{22}^{\epsilon}\left(T, q^{\epsilon}\right)}\right| \\
& +\left|\frac{\psi_{11}^{\epsilon}\left(T, q^{\epsilon}\right) h_{\sigma}^{0}\left(q^{\epsilon}\right)+\psi_{12}^{\epsilon}\left(T, q^{\epsilon}\right)}{\psi_{21}^{\epsilon}\left(T, q^{\epsilon}\right) h_{\sigma}^{0}\left(q^{\epsilon}\right)+\psi_{22}^{\epsilon}\left(T, q^{\epsilon}\right)}-\frac{\psi_{11}^{0}\left(T, q^{0}\right) h_{\sigma}^{0}\left(q^{0}\right)+\psi_{12}^{0}\left(T, q^{0}\right)}{\psi_{21}^{0}\left(T, q^{0}\right) h_{\sigma}^{0}\left(q^{0}\right)+\psi_{22}^{0}\left(T, q^{0}\right)}\right| \\
:= & I+I I .
\end{aligned}
$$

To estimate the quantities $I$ and $I I$, let us consider, for real numbers $\xi$ and $2 \times 2$ matrices $D=\left(d_{i j}\right)$, the function $u: \mathbb{R} \times \mathbb{R}^{4} \rightarrow \mathbb{R}$ defined by

$$
u(\xi, D)=\frac{d_{11} \xi+d_{12}}{d_{21} \xi+d_{22}} .
$$


Using this function, we have

$$
\begin{gathered}
I=\left|u\left(y, \Psi^{\epsilon}\left(T, q^{\epsilon}\right)\right)-u\left(x, \Psi^{\epsilon}\left(T, q^{\epsilon}\right)\right)\right|, \\
I I=\left|u\left(x, \Psi^{\epsilon}\left(T, q^{\epsilon}\right)\right)-u\left(z, \Psi^{0}\left(T, q^{0}\right)\right)\right|,
\end{gathered}
$$

where $x:=h_{\sigma}^{0}\left(q^{\epsilon}\right), y:=h_{\sigma}^{\epsilon}\left(q^{\epsilon}\right)$, and $z:=h_{\sigma}^{0}(q)$.

To estimate $I$, apply the mean value theorem to the function $\xi \mapsto u(\xi, D)$ and use the fact that

$$
u_{\xi}(\xi, D)=\frac{\operatorname{det} D}{d_{21} \xi+d_{22}}
$$

to obtain the inequality

$$
I \leq\left|\frac{\operatorname{det} \Psi^{\epsilon}\left(T, q^{\epsilon}\right)}{\psi_{21}^{\epsilon}\left(T, q^{\epsilon}\right) \xi+\psi_{22}^{\epsilon}\left(T, q^{\epsilon}\right)}\right|\left|h_{\sigma}^{\epsilon}\left(q^{\epsilon}\right)-h_{\sigma}^{0}\left(q^{\epsilon}\right)\right|
$$

for some $\xi$ between $h_{\sigma}^{\epsilon}\left(q^{\epsilon}\right)$ and $h_{\sigma}^{0}\left(q^{\epsilon}\right)$. Let us note that $\left|\xi-h_{\sigma}^{0}\left(q^{\epsilon}\right)\right|<r$. Also, if $\mu>0$ is sufficiently small, then $\left|\Psi^{\epsilon}\left(T, q^{\epsilon}\right)-\Psi^{0}\left(T, q^{0}\right)\right|_{C^{0}}<r$. Thus, if we use the inequalities (8.27) together with a triangle law estimate for the term containing the determinant, then we find that $I \leq \lambda\left|h_{\sigma}^{\epsilon}-h_{\sigma}^{0}\right|_{C^{0}}$ for $\lambda=27 / 32<1$.

To estimate $I I$, let us note that the function $u$ is Lipschitz on the set

$$
\left\{(\xi, D): \xi=h_{\sigma}^{0}(q), D=\Psi^{\epsilon}(T, q), q \in \mathbb{S}^{1}, 0 \leq \epsilon<\epsilon^{*}\right\} .
$$

In particular, there is a constant $L_{1}>0$ such that

$$
I I \leq L_{1}\left(\left|h_{\sigma}^{0}\left(q^{\epsilon}\right)-h_{\sigma}^{0}\left(q^{0}\right)\right|+\left|\Psi^{\epsilon}\left(T, q^{\epsilon}\right)-\Psi^{0}\left(T, q^{0}\right)\right|\right) .
$$

Using the fact that $h^{0}$ is Lipschitz on $\mathbb{S}^{1}$, we conclude that there exist constants $L>0$ and $C_{4}>0$ such that

$$
I I \leq L\left(\left|q^{\epsilon}-q^{0}\right|+\left|\Psi^{\epsilon}\left(T, q^{\epsilon}\right)-\Psi^{0}\left(T, q^{0}\right)\right|\right) \leq C_{4} \epsilon .
$$

Thus,

$$
\left|h_{\sigma}^{\epsilon}(p)-h_{\sigma}^{0}(p)\right| \leq C_{4} \epsilon+\lambda\left|h_{\sigma}^{\epsilon}(q)-h_{\sigma}^{0}(q)\right| \leq C_{4} \epsilon+\lambda\left|h_{\sigma}^{\epsilon}-h_{\sigma}^{0}\right|_{C^{0}},
$$

and, as a result,

$$
\left|h_{\sigma}^{\epsilon}-h_{\sigma}^{0}\right|_{C^{0}} \leq \frac{C_{4}}{1-\lambda} \epsilon .
$$

This completes the proof of the claim.

In addition to the restrictions on the size of $\mu$ already made, let us also require that $\mu<r / C$, where $C$ is the constant appearing in the claim. Define

$$
\epsilon_{0}=\sup \left\{\epsilon^{\prime}: 0 \leq \epsilon^{\prime}<\epsilon_{*},\left|h_{\sigma}^{\epsilon}-h_{\sigma}^{0}\right|_{C^{0}} \leq r \text { for } \epsilon \in\left[0, \epsilon^{\prime}\right]\right\} .
$$

We will show that $\epsilon_{0}=\epsilon_{*}$. Suppose not, then $\epsilon_{0}<\epsilon_{*}$. For $\epsilon<\epsilon_{0},\left|h_{\sigma}^{\epsilon}-h_{\sigma}^{0}\right|_{C^{0}} \leq r$ and, hence, $\left|h_{\sigma}^{\epsilon}-h_{\sigma}^{0}\right|_{C^{0}} \leq C \epsilon$ by the claim. Since $\epsilon_{0}<\epsilon_{*}$, the graph of $h^{\epsilon_{0}}$ is normally hyperbolic by the definition of $\epsilon_{*}$. Passing to the limit as $\epsilon \rightarrow \epsilon_{0}$, we have $\left|h_{\sigma}^{\epsilon_{0}}-h_{\sigma}^{0}\right|_{C^{0}} \leq C \epsilon_{0}<r$. This contradicts the fact that $\epsilon_{0}$ is the supremum; hence $\epsilon_{0}=\epsilon_{*}$. Now, by the claim, we conclude that $\left|h_{\sigma}^{\epsilon}-h_{\sigma}^{0}\right|_{C^{0}} \leq C \epsilon$ for $0 \leq \epsilon<\epsilon_{*}$. 
Let us now estimate $h_{\tau}^{\epsilon}$. Note first that the invariance of the graph of the function $h^{\epsilon}$ is equivalent to the identities

$$
\begin{aligned}
h_{\sigma}^{\epsilon} \sigma^{\prime}+h_{\tau}^{\epsilon} & =f\left(h^{\epsilon}, \sigma\right)+\epsilon R\left(h^{\epsilon}, \sigma, \tau / \mu^{2}, \mu\right), \\
\sigma^{\prime} & =g\left(h^{\epsilon}, \sigma\right)+\epsilon S\left(h^{\epsilon}, \sigma, \tau / \mu^{2}, \mu\right) .
\end{aligned}
$$

If we suppose that $h^{\epsilon}(\sigma, \tau)=h^{0}(\sigma)+\epsilon H(\sigma, \tau, \epsilon)$ and substitute this expression into the relation (8.29), then we obtain the equation

$$
\begin{aligned}
\left(g\left(h^{0}+\epsilon H, \sigma\right)\right. & \left.+\epsilon S\left(h^{0}+\epsilon H, \sigma, \tau / \mu^{2}, \mu\right)\right) H_{\sigma}+H_{\tau} \\
& =\frac{1}{\epsilon}\left(f\left(h^{0}+\epsilon H, \sigma\right)-h_{\sigma}^{0} g\left(h^{0}+\epsilon H, \sigma\right)\right)-h_{\sigma}^{0} S+R .
\end{aligned}
$$

Finally, using the estimate

$$
\left|H_{\sigma}\right|_{C^{0}}=\frac{1}{\epsilon}\left|h_{\sigma}^{\epsilon}-h_{\sigma}^{0}\right|_{C^{0}} \leq C_{1}
$$

and the relation $f\left(h^{0}, \sigma\right)=h_{\sigma}^{0} g\left(h^{0}, \sigma\right)$, we conclude that there is a constant $C>0$, that is independent of $\epsilon$, such that $\left|H_{\tau}\right|_{C^{0}} \leq C$; that is, $\left|h_{\tau}^{\epsilon}\right|_{C^{0}} \leq C \epsilon$.

Let us note that we will eventually have to verify estimates as in display (8.11). As a step in this direction, let us first consider $\lambda_{3}$ and note, from the formula (8.15), that the growth estimate requires an asymptotic estimate of the norm of $X_{2}(s)$. By the definition of $X_{2}(s)$, it is clear that this norm estimate is determined by the behavior of the function $s \mapsto y^{\epsilon}(s, q)$ defined in display (8.8). To determine this behavior, we must estimate the integral

$$
\int_{0}^{s}\left(G_{\rho} h_{\sigma}^{\epsilon}+G_{\sigma}\right) d t
$$

The precise integral estimate that we will require is the content of the next lemma.

LEMma 8.4. If, for all $s \geq 0$, the function $s \mapsto G\left(h^{\epsilon}(\sigma(s), \tau(s)), \sigma(s), \tau(s), \epsilon\right)$ has no zeros, and if $\mu>0$ is sufficiently small, then there is a constant $C>0$ such that

$$
y^{\epsilon}(s, q)=\exp \left(\int_{0}^{s}\left(G_{\rho} h_{\sigma}^{\epsilon}+G_{\sigma}\right) d t\right) \geq e^{-C} e^{-C \epsilon s} .
$$

Proof. Note that

$$
\begin{aligned}
\frac{d}{d s} \ln \left|G\left(h^{\epsilon}(\sigma(s), \tau(s)), \sigma(s), \tau(s), \epsilon\right)\right| & =\frac{1}{G}\left(G_{\rho} h_{\sigma}^{\epsilon} \sigma^{\prime}+G_{\rho} h_{\tau}^{\epsilon}+G_{\sigma} \sigma^{\prime}+G_{\tau}\right) \\
& =G_{\rho} h_{\sigma}^{\epsilon}+G_{\sigma}+\frac{G_{\rho} h_{\tau}^{\epsilon}}{G}+\frac{G_{\tau}}{G} .
\end{aligned}
$$

After integration over the interval $[0, s]$ and a rearrangement, we obtain the identity

$$
\begin{aligned}
\int_{0}^{s}\left(G_{\rho} h_{\sigma}^{\epsilon}+G_{\sigma}\right) d t=\ln & \left|\frac{G\left(h^{\epsilon}(\sigma(s), \tau(s)), \sigma(s), \tau(s), \epsilon\right)}{G\left(h^{\epsilon}(\sigma(0), \tau(0)), \sigma(0), \tau(0), \epsilon\right)}\right| \\
& -\int_{0}^{s} \frac{G_{\rho} h_{\tau}^{\epsilon}}{G} d t-\int_{0}^{s} \frac{G_{\tau}}{G} d t .
\end{aligned}
$$


Recall the definition (8.1) of $G$, and note that

$$
\begin{aligned}
& \frac{d}{d s}\left(\frac{S}{G}\right)=\frac{S_{\rho} h_{\sigma}^{\epsilon} \sigma^{\prime}+S_{\rho} h_{\tau}^{\epsilon}+S_{\sigma} \sigma^{\prime}+\frac{1}{\mu^{2}} S_{\tau}}{G} \\
& -\frac{S\left(G_{\rho} h_{\sigma}^{\epsilon} \sigma^{\prime}+G_{\rho} h_{\tau}^{\epsilon}+G_{\sigma} \sigma^{\prime}+\frac{\epsilon}{\mu^{2}} S_{\tau}\right)}{G^{2}} .
\end{aligned}
$$

Using this identity and an easy computation, we find that

$$
\begin{aligned}
\int_{0}^{s} \frac{G_{\tau}}{G} d t=\frac{\epsilon}{\mu^{2}} \int_{0}^{s} \frac{S_{\tau}}{G} d t & \\
=\epsilon\left(\frac{S\left(h^{\epsilon}(\sigma(s), \tau(s)), \sigma(s), \tau(s), \epsilon\right)}{G\left(h^{\epsilon}(\sigma(s), \tau(s)), \sigma(s), \tau(s), \epsilon\right)}-\frac{S\left(h^{\epsilon}(\sigma(0), \tau(0)), \sigma(0), \tau(0), \epsilon\right)}{G\left(h^{\epsilon}(\sigma(0), \tau(0)), \sigma(0), \tau(0), \epsilon\right)}\right) & \\
& \quad-\epsilon \int_{0}^{s} \frac{S_{\rho} h_{\sigma}^{\epsilon} G+S_{\rho} h_{\tau}^{\epsilon}+S_{\sigma} G}{G} d t \\
& \quad+\epsilon \int_{0}^{s} \frac{S G_{\rho} h_{\sigma}^{\epsilon} G+S G_{\rho} h_{\tau}^{\epsilon}+S G_{\sigma} G}{G^{2}} d t+\frac{\epsilon^{2}}{\mu^{2}} \int_{0}^{s} \frac{S S_{\tau}}{G^{2}} d \tau .
\end{aligned}
$$

Note that, because their integrands are bounded, all terms except the last one on the right-hand side of the final equality of display $(8.32)$ are $O(\epsilon)$. To estimate the last term, let us differentiate the function $S^{2} / G^{2}$ with respect to $s$, and rearrange the resulting identity, to obtain the following expression:

$$
\begin{aligned}
\frac{S S_{\tau}}{G^{2}}= & \frac{\mu^{2}}{2} \frac{d}{d s}\left(\frac{S^{2}}{G^{2}}\right)-\mu^{2} S \frac{S_{\rho} h_{\sigma}^{\epsilon} G+S_{\rho} h_{\tau}^{\epsilon}+S_{\sigma} G}{G^{2}} \\
& +\mu^{2} S^{2} \frac{G_{\rho} h_{\sigma}^{\epsilon} G+G_{\rho} h_{\tau}^{\epsilon}+G_{\sigma} G}{G^{3}}+\epsilon S^{2} \frac{S_{\tau}}{G^{3}} .
\end{aligned}
$$

If we integrate both sides of the last identity over the interval $[0, s]$, then all the integrands are bounded. In view of this fact and the inequality $\epsilon \leq \mu$, it follows that

$$
\frac{\epsilon^{2}}{\mu^{2}} \int_{0}^{s} \frac{S S_{\tau}}{G^{2}} d t=s O(\epsilon)
$$

To estimate the term

$$
\int_{0}^{s} \frac{G_{\rho} h_{\tau}^{\epsilon}}{G} d t
$$

that appears in the expression (8.31) for the integral

$$
\int_{0}^{s}\left(G_{\rho} h_{\sigma}^{\epsilon}+G_{\sigma}\right) d t
$$

use the inequality $\left|h_{\tau}^{\epsilon}\right|_{C^{0}} \leq C \epsilon$ obtained in Lemma 8.3.

In summary, we have $\left|\int_{0}^{s}\left(G_{\rho} h_{\sigma}^{\epsilon}+G_{\sigma}\right) d t\right| \leq C_{1}+C_{2} \epsilon s$ for some constants $C_{1}>0$ and $C_{2}>0$ both independent of $\epsilon$.

LEMma 8.5. With the hypotheses of Lemma 8.3, if $\mu>0$ is sufficiently small, then there is a constant $C>0$ such that $\left|h_{\sigma \sigma}^{\epsilon}\right|_{C^{0}} \leq C$.

Proof. The proof of the lemma is similar to the proof of Lemma 8.3. 
Recall that $h_{\sigma}^{\epsilon}$ satisfies the "fixed point equation" in display (8.23), and choose $\mu>0$ sufficiently small so that $T(\mu)$ is as in the proof of Lemma 8.3. If we set $p=\sigma\left(T, q^{\epsilon}\right)$ and $s=T$, then we have the identity

$$
h_{\sigma}^{\epsilon}(p)=\frac{\psi_{11}^{\epsilon}\left(T, q^{\epsilon}\right) h_{\sigma}^{\epsilon}\left(q^{\epsilon}\right)+\psi_{12}^{\epsilon}\left(T, q^{\epsilon}\right)}{\psi_{21}^{\epsilon}\left(T, q^{\epsilon}\right) h_{\sigma}^{\epsilon}\left(q^{\epsilon}\right)+\psi_{22}^{\epsilon}\left(T, q^{\epsilon}\right)} .
$$

By a direct computation of the derivative of both sides of (8.33) with respect to $p$, we obtain

$$
\begin{aligned}
h_{\sigma \sigma}^{\epsilon}(p)= & \frac{\frac{d q^{\epsilon}}{d p}}{\left(\psi_{21}^{\epsilon} h_{\sigma}^{\epsilon}\left(q^{\epsilon}\right)+\psi_{22}^{\epsilon}\right)^{2}}\left(\left(\psi_{21}^{\epsilon} \frac{d}{d q} \psi_{11}^{\epsilon}-\psi_{11}^{\epsilon} \frac{d}{d q} \psi_{21}^{\epsilon}\right)\left(h_{\sigma}^{\epsilon}\left(q^{\epsilon}\right)\right)^{2}\right. \\
& +\left(\psi_{22}^{\epsilon} \frac{d}{d q} \psi_{11}^{\epsilon}-\psi_{12}^{\epsilon} \frac{d}{d q} \psi_{21}^{\epsilon}+\psi_{21}^{\epsilon} \frac{d}{d q} \psi_{12}^{\epsilon}-\psi_{11}^{\epsilon} \frac{d}{d q} \psi_{22}^{\epsilon}\right) h_{\sigma}^{\epsilon}\left(q^{\epsilon}\right) \\
& \left.+\left(\psi_{11}^{\epsilon} \psi_{22}^{\epsilon}-\psi_{12}^{\epsilon} \psi_{21}^{\epsilon}\right) h_{\sigma \sigma}^{\epsilon}\left(q^{\epsilon}\right)+\psi_{22}^{\epsilon} \frac{d}{d q} \psi_{12}^{\epsilon}-\psi_{12}^{\epsilon} \frac{d}{d q} \psi_{22}^{\epsilon}\right),
\end{aligned}
$$

where the functions $\psi_{i j}^{\epsilon}, i, j=1,2$, on the right-hand side of the equality are evaluated at $\left(T, q^{\epsilon}\right)$.

Using the identity $p=\sigma^{\epsilon}\left(T, q^{\epsilon}\right)$ and differentiating with respect to $p$, we have that $d q^{\epsilon} / d p=1 / \sigma_{q}^{\epsilon}\left(T, q^{\epsilon}\right)$. Moreover, using the solution (8.4) and Proposition 8.1, it is not difficult to see that $\sigma_{q}^{\epsilon}(T, q)=y^{\epsilon}(T, q)$, where, $y^{\epsilon}$ is defined in Proposition 8.1. If $\mu>0$ is sufficiently small so that $h_{\sigma}^{\epsilon}$ is sufficiently close to $h_{\sigma}^{0}$, the matrix $\Psi^{\epsilon}(T, \cdot)$ is sufficiently close to $\Psi^{0}\left(T_{0}, \cdot\right)$, and the partial derivative $\Psi_{q}^{\epsilon}$ is uniformly bounded, then, for all $q$ and all $T=T(\mu)$, we have

$$
\left|\operatorname{det} \Psi^{\epsilon}(T, q)\right|<\frac{1}{4} M_{0}^{2}, \quad\left|\left(\psi_{21}^{\epsilon}(T, q) h_{\sigma}^{\epsilon}(q)+\psi_{22}^{\epsilon}(T, q)\right)^{2}\right|>\frac{1}{3} M_{0},
$$

where $M_{0}>0$ is the constant appearing in Lemma 8.3. Thus, using Lemma 8.4, the absolute value of the coefficient

$$
\frac{\frac{d q^{\epsilon}}{d p}}{\left(\psi_{21}^{\epsilon} h_{\sigma}^{\epsilon}\left(q^{\epsilon}\right)+\psi_{22}^{\epsilon}\right)^{2}}\left(\psi_{11}^{\epsilon} \psi_{22}^{\epsilon}-\psi_{12}^{\epsilon} \psi_{21}^{\epsilon}\right)
$$

will be uniformly bounded less than one.

To estimate the supremum of $h_{\sigma \sigma}$, we proceed in the following order: We take the absolute value of each side of (8.34), apply the triangle law to the right-hand side, take the supremum of the right-hand side over $q^{\epsilon}$, take the supremum of the left-hand side over $p$, move the term containing the norm of $h_{\sigma \sigma}$ on the right-hand side to the left-hand side, collect terms, and then divide both sides by the coefficient of the norm of $h_{\sigma \sigma}$. This coefficient is not zero because of the uniform bound on the absolute value of the quantity in display (8.35). Thus, we obtain a uniform bound on the norm of $h_{\sigma \sigma}$.

9. Proof of Lemma 7.3. If $\mu>0$ is chosen as in Lemma 8.3 and $\epsilon_{*}>0$ is such that, for $0 \leq \epsilon<\epsilon_{*}$, the system $E^{\epsilon, \mu}$ has a $k$-normally hyperbolic invariant manifold, $k \geq 2$, that is the graph of a $C^{k}$ function $h^{\epsilon}$ of the angular variables, then, by Lemmas 8.3 and 8.5, the subset $\mathcal{S}:=\left\{h^{\epsilon}: 0 \leq \epsilon<\epsilon_{*}\right\}$ in the space of $C^{2}$ functions of the angular variables is uniformly bounded. As a result, the set $\mathcal{S}$ is equicontinuous in the $C^{1}$ norm. By Arzela's theorem, if we choose a sequence of real 
numbers increasing to the limit $\epsilon_{*}$, then we can extract a subsequence $\left\{\epsilon_{k}\right\}$ such that the corresponding sequence $\left\{h^{\epsilon_{k}}\right\}$ converges to a $C^{1}$ function $h^{\epsilon_{*}}$. An easy argument, as in Lemma 5.3, shows that the graph of $h^{\epsilon_{*}}$ is invariant under the flow of $E^{\epsilon_{*}, \mu}$, as required.

10. Proof of Lemma 7.4. If $\mu>0$ is chosen as in Lemma 8.3, the number $\epsilon_{*}>0$ is such that, for $0 \leq \epsilon<\epsilon_{*}$, the system $E^{\epsilon, \mu}$ has a $k$-normally hyperbolic invariant manifold that is the graph of a $C^{k}$ function $h^{\epsilon}$ of the angular variables, and if $E^{\epsilon_{*}, \mu}$ has a $C^{1}$ invariant manifold $M\left(\epsilon_{*}, \mu\right)$ given as the graph of the function $h^{\epsilon_{*}}$ of the angular variables, then we must show that $M\left(\epsilon_{*}, \mu\right)$ has a continuous invariant normal bundle.

It suffices to construct a normal bundle over the curve

$$
q \mapsto\left(h^{\epsilon_{*}}(q, 0), q, 0\right)
$$

that is invariant with respect to some iterate of the stroboscopic linearized Poincaré map; that is, the map given by moving a point on the slice $\{(\rho, \sigma, \tau): \tau=0\}$ forward by the flow to time $2 \pi m \mu^{2} / \Omega$. To prove this reduction, note that the linearized Poincaré map is two-dimensional at each point and that the tangent line to the invariant torus is invariant under the map. Also, for a two-dimensional linear map with an invariant line, if an iterate of the map has two distinct invariant lines, then so does the map. Finally, if there is a normal bundle over the curve, then a normal bundle over the torus is constructed by moving the vectors in the given normal bundle forward by the linearized flow.

We will construct a normal bundle over the curve (10.1). For this, let us consider the function space

$$
\Gamma:=\left\{\alpha: \mathbb{S}^{1} \rightarrow \mathbb{R}: \alpha \in C^{0}\right\} .
$$

Also, for $\alpha \in \Gamma$, let us define a vector at the point $\left(h^{\epsilon_{*}}(q, 0), q, 0\right)$ as follows:

$$
X_{0}(q):=\alpha(q) X_{2}(q)+X_{2}(q)^{\perp},
$$

where $X_{2}(q):=\mathcal{X}_{2}\left(h^{\epsilon_{*}}(q, 0), q, 0\right)$. We will show that there is some choice for $\alpha \in \Gamma$ so that $X_{0}$ generates an invariant normal bundle over the curve (10.1).

If $n$ is an integer, $T:=2 \pi m n \mu^{2} / \Omega$, and $p=\sigma^{\epsilon_{*}}(T, q)$, then $X_{0}$ generates an invariant bundle if and only if

$$
\lambda^{\epsilon_{*}}(T, q) X_{0}(p)=\left(\alpha(q)+a^{\epsilon_{*}}(T, q)\right) X_{2}(p)+b^{\epsilon_{*}}(T, q) X_{2}(p)^{\perp},
$$

where $\lambda^{\epsilon_{*}}, a^{\epsilon_{*}}$, and $b^{\epsilon_{*}}$ are defined in subsection 8.2 for the system corresponding to $\epsilon_{*}$. Using these definitions, we find that (10.2) holds if and only if

$$
\alpha(p)=\frac{\alpha(q)+a^{\epsilon_{*}}(T, q)}{b^{\epsilon_{*}}(T, q)} .
$$

Define $T<0$ analogous to the definition in display (8.24) with the property that, for $0 \leq \epsilon<\epsilon_{*}$, there is some $\eta$ such that

$$
b^{\epsilon}(T):=\sup \left\{b^{\epsilon}(T, q): q \in \mathbb{S}^{1}\right\}>\eta>1 .
$$

Passing to the limit as $\epsilon$ approaches $\epsilon_{*}$ from below and using the fact that $h^{\epsilon}$ converges to $h^{\epsilon_{*}}$, we find that

$$
b^{\epsilon_{*}}(T):=\sup \left\{b^{\epsilon_{*}}(T, q): q \in \mathbb{S}^{1}\right\} \geq \eta>1
$$


Also, let us define $\Lambda: \Gamma \rightarrow \Gamma$ by

$$
(\Lambda \alpha)(p)=\frac{\alpha(q)+a^{\epsilon_{*}}(T, q)}{b^{\epsilon_{*}}(T, q)} .
$$

A fixed point of $\Lambda$ corresponds to the desired invariant normal bundle. But, by a simple computation, we have the inequality

$$
\left|\left(\Lambda \alpha_{2}\right)(p)-\left(\Lambda \alpha_{1}\right)(p)\right| \leq \frac{1}{\eta}\left|\alpha_{2}(q)-\alpha_{1}(q)\right| .
$$

Thus, $\Lambda$ is a contraction on the complete metric space $\Gamma$ and $\Lambda$ has a unique fixed point, as required.

11. Proof of Lemma 7.5. We will show that the $C^{1}$ invariant manifold given as the graph of the function $h^{\epsilon_{*}}$ is $k$-normally hyperbolic under the assumption that this manifold has an invariant normal splitting. For this, we must verify the inequalities given in display (8.11).

Consider $\lambda_{3}(s)$, and recall formula (8.15). Let us suppose that a bounded neighborhood $N$ as in Proposition 6.2 is chosen, the invariant tori are given by the graphs of the functions $h^{\epsilon}$ of the angular variables, and $\mu>0$ is sufficiently small so that the invariant manifold given by $h^{\epsilon_{*}}$ is in $N$. Then, by Lemma 8.3, the functions $h^{\epsilon}$ satisfy the inequality

$$
\left|h^{\epsilon}-h^{0}\right|<C \epsilon
$$

for $0 \leq \epsilon \leq \epsilon_{*}$.

Let $s \rightarrow \gamma^{\epsilon_{*}}(s, q, \tau)=\left(h^{\epsilon_{*}}\left(\sigma^{\epsilon_{*}}(s, q, \tau), s+\tau\right), \sigma^{\epsilon_{*}}(s, q, \tau), s+\tau\right)$ be the solution of (7.3) corresponding to $\epsilon_{*}$ with the initial condition $\left(h^{\epsilon_{*}}(q, \tau), q, \tau\right)$, let $B$ be the system matrix of the linearization of system (7.3) along the solution $\gamma^{\epsilon}$, and note that

$$
\operatorname{tr} B\left(\gamma^{\epsilon_{*}}(t, q, \tau)\right)=f_{\rho}+g_{\sigma}+\epsilon\left(R_{\rho}+S_{\sigma}\right) .
$$

Let $\omega$ be the minimal period of the periodic solution of the unperturbed system $(7.2)$, let $(\bar{q}, \bar{\tau})$ be an arbitrary choice of the angular variables, and define

$$
b:=\int_{0}^{\omega} \operatorname{tr} B\left(\gamma^{0}(s, \bar{q}, \bar{\tau})\right) d s .
$$

The quantity $b$ is a Floquet exponent of the periodic orbit that is independent of the choice of the angular variables. By an application of Gronwall's inequality (8.16), there is a constant $C_{1}>0$ such that, for $0 \leq s \leq \omega$,

$$
\left|\gamma^{\epsilon_{*}}(s, \bar{q}, \bar{\tau})-\gamma^{0}(s, \bar{q}, \bar{\tau})\right| \leq C_{1} \epsilon_{*}
$$

Hence, there is a constant $C_{2}>0$ such that

$$
\left|\operatorname{tr} B\left(\gamma^{\epsilon_{*}}(s, \bar{q}, \bar{\tau})\right)-\operatorname{tr} B\left(\gamma^{0}(s, \bar{q}, \bar{\tau})\right)\right| \leq C_{2} \epsilon_{*}
$$

and

$$
\int_{0}^{\omega} \operatorname{tr} B\left(\gamma^{\epsilon_{*}}(s, \bar{q}, \bar{\tau})\right) d s \leq \int_{0}^{\omega} \operatorname{tr} B\left(\gamma^{0}(s, \bar{q}, \bar{\tau})\right) d s+C_{2} \epsilon_{*} \omega=b+C_{2} \epsilon_{*} \omega .
$$


An arbitrary $s \geq 0$ can be expressed in the form $s=\ell \omega+r$ with $0 \leq r<\omega$. Also, for $k=1,2, \ldots, \ell$, let us define

$$
q_{k}:=\sigma^{\epsilon_{*}}(k \omega, q, \tau), \quad \tau_{k}:=k \omega+\tau .
$$

There are constants $C_{3}>0$ and $C_{4}>0$ such that

$$
\begin{aligned}
& \int_{0}^{s} \operatorname{tr} B\left(\gamma^{\epsilon_{*}}(t, q, \tau)\right) d t= \sum_{k=0}^{\ell-1} \int_{k \omega}^{(k+1) \omega} \operatorname{tr} B\left(\gamma^{\epsilon_{*}}(t, q, \tau)\right) d t \\
& \quad+\int_{\ell \omega}^{\ell \omega+r} \operatorname{tr} B\left(\gamma^{\epsilon_{*}}(t, q, \tau)\right) d t \\
&= \sum_{k=0}^{\ell-1} \int_{0}^{\omega} \operatorname{tr} B\left(\gamma^{\epsilon_{*}}\left(t, q_{k}, \tau_{k}\right)\right) d t \\
&+\int_{0}^{r} \operatorname{tr} B\left(\gamma^{\epsilon_{*}}\left(t, q_{\ell}, \tau_{\ell}\right)\right) d t \\
& \leq \ell\left(b+C_{2} \epsilon_{*} \omega\right)+C_{3} \leq\left(\frac{b}{\omega}+C_{2} \epsilon_{*}\right) s+C_{4} .
\end{aligned}
$$

By Proposition 8.1 and Lemma 8.4, there are constants $C_{5}>0$ and $C_{6}>0$ such that

$$
\left|X_{2}(s)\right| \geq C_{6} e^{-\mu C_{5} s} .
$$

Also, by Lemma 7.4, the function $\alpha$ corresponding to the normal splitting at $\epsilon_{*}$ is bounded as a periodic function over the invariant manifold.

Taking the above estimates into account and using formula (8.15), there is a constant $c>0$ such that $\lambda_{3}(s) \leq c e^{\left(\frac{b}{\omega}+c \mu\right) s}$. If, in addition, $\mu>0$ is sufficiently small, then $-\beta:=\frac{b}{\omega}+c \mu<0$, and we have the desired estimate:

$$
\lambda_{3}(s) \leq c e^{-\beta s}
$$

for all $s \geq 0$.

The function $\left|X_{1}(s)\right|$ is uniformly bounded below, in fact $\left|X_{1}(s)\right| \geq 1$. Thus, if $k$ is a positive integer, then using the estimate (11.3), there is some $c_{1}>0$ such that

$$
\frac{\lambda_{3}(s)}{\lambda_{1}^{k}(s)} \leq c_{1} e^{-\beta s} .
$$

Using the estimates (11.2) and (11.3), we have that

$$
\frac{\lambda_{3}(s)}{\lambda_{1}^{k}(s)} \leq \frac{c_{1}}{C_{6}^{k}} e^{-s\left(\beta-\mu k c_{1}\right)}
$$

Thus, if $\mu>0$ is sufficiently small, there are constants $c_{2}>0$ and $\beta_{1}>0$ such that

$$
\frac{\lambda_{3}(s)}{\lambda_{2}^{k}(s)} \leq c_{2} e^{-\beta_{1} s} .
$$

Finally, using the general smoothness result in [7], it follows that the $C^{1}$ manifold given as the graph of $h^{\epsilon_{*}}$ with invariant splitting and with the hyperbolic estimates just proved is in fact a $C^{k}$ manifold, as required. 
12. Appendix. In this appendix we will prove Propositions 6.1 and 6.2.

Proof of Proposition 6.1. We will construct a family of $C^{1}$ curves $S_{r}^{+}$and $S_{r}^{-}$, $r \in(0,1]$ for system $(6.1)$ such that the following properties are satisfied:

(i) The curves $S_{r}^{+}$and $S_{r}^{-}$lie in the exterior and interior domains separated by $\Gamma$, respectively, and $S_{r}^{+}$(resp., $S_{r}^{-}$) together with $\Gamma$ encloses an annulus.

(ii) The curves $S_{r}^{+}$and $S_{r}^{-}$are transverse to the vector field $f$.

(iii) There is a constant $C_{0}>0$ that is independent of $r$ such that $\sup \{d(x, \Gamma)$ : $\left.x \in S_{r}^{ \pm}\right\} \leq C_{0} r$.

(iv) If $C_{f}^{ \pm}(r):=\min _{x \in S_{r}^{ \pm}}\{\langle f(x), n(x)\rangle\}$, where $n(x)$ is the inward (resp., outward) unit normal vector to $S_{r}^{+}$(resp., $S_{r}^{-}$) at $x \in S_{r}^{ \pm}$and the angle brackets denote the usual inner product, then $C_{f}^{ \pm}(r) \geq C_{1} r$ for some constant $C_{1}>0$ independent of $r$.

Let us assume for the moment that the above construction is possible and use it to complete the proof of the proposition.

For this, let $N \subset \mathbb{R}^{2}$ be the annulus such that $\partial N=S_{1}^{+} \cup S_{1}^{-}$. If $\|g\|_{C^{0}}$ is small enough, then there exists an $r_{0} \in(0,1]$ such that $\|g\|_{C^{0}}=C_{1} r_{0}$. Using this fact, we have, for $r>r_{0}$ and $x \in S_{r}^{ \pm}$, that

$$
\langle f(x)+g(x), n(x)\rangle \geq C_{f}^{ \pm}(r)-\|g\|_{C^{0}} \geq C_{1} r-C_{1} r_{0}>0 .
$$

Thus, for $r>r_{0}$, the set $S_{r}^{+} \cup S_{r}^{-}$encloses a positively invariant annulus for the system (6.2) in $\mathbb{R}^{2}$. It follows that $\bar{\Gamma}$ is contained in this domain, and, by the estimates given in (iii) and (iv), that $d(x, \Gamma) \leq C_{0} r_{0}=\frac{C_{0}}{C_{1}}\|g\|_{C^{0}}$ for every $x \in \bar{\Gamma}$, as required.

The proof will be completed by constructing a family of curves that satisfies properties (i)-(iv).

The constructions and the verifications of properties (i)-(iv) for the families $S_{r}^{+}$ and $S_{r}^{-}$are similar. We will give the proof for $S_{r}^{+}$only. Also, in the arguments to follow we will suppress the superscript "+."

\section{Step 1. Construction of $S_{r}$.}

Since the periodic solution $\Gamma$ is asymptotically stable, there exists a neighborhood of $\Gamma$, contained in the stable manifold of $\Gamma$, with an invariant foliation with respect to the system (6.1) whose leaves are curves. Let $M^{s}(p)$ denote the leaf through the point $p \in \Gamma$. Also, let $t \mapsto x(t, \xi)$ denote the solution of the differential equation (6.1) with $x(0, \xi)=\xi$, and let $\Phi(t, \xi)$ denote the principal fundamental matrix solution at $t=0$ of the linearized system along this solution.

Fix a point $q_{1} \in M^{s}(p)$ that lies in the exterior domain separated by $\Gamma$, and let $q_{0}:=x\left(\omega, q_{1}\right)$ be the point where the solution through $q_{1}$ first returns to $M^{s}(p)$. Choose a smooth function $q:[0,1] \rightarrow M^{s}(p) \subset \mathbb{R}^{2}$ such that the derivative of $q$, including the left-hand and right-hand derivatives at the end points of its domain, does not vanish, and with the additional properties that $q(0)=q_{0}, q(1)=q_{1}$, and

$$
\dot{q}\left(0^{+}\right)=\Phi\left(\omega, q_{1}\right) \dot{q}\left(1^{-}\right) .
$$

The last requirement can be met because, by the invariance of the foliation,

$$
\Phi\left(\omega, q_{1}\right) T_{q_{1}} M^{s}(p)=T_{q_{0}} M^{s}(p) .
$$

Let $t:[0,1] \rightarrow[0, \omega]$ be the linear transformation given by $t(\lambda)=\lambda \omega$, and consider the curve $S$ defined parametrically by $\lambda \mapsto x(t(\lambda), q(\lambda))$. Let us note that $S$ is closed. Indeed, since $t(0)=0$ and $t(1)=\omega$, we have that $x(t(0), q(0))=x(t(1), q(1))=q_{0}$. 
For each $\lambda \in(0,1)$, define $T(\lambda)$ to be the tangent vector to $S$ at the point $x(t(\lambda), q(\lambda))$ given by

$$
\begin{aligned}
T(\lambda) & =\left.\frac{d}{d s} x(t(s), q(s))\right|_{s=\lambda}=\omega \dot{x}(t(\lambda), q(\lambda))+x_{\xi}(t(\lambda), q(\lambda)) \dot{q}(\lambda) \\
& =\omega f(x(t(\lambda), q(\lambda)))+\Phi(t(\lambda), q(\lambda)) \dot{q}(\lambda) .
\end{aligned}
$$

To check that $S$ is a $C^{1}$ curve, it suffices to show that $T\left(0^{+}\right)=T\left(1^{-}\right)$. But, this equality follows from the identities (12.1) and (12.2).

Let $\phi^{s}$ denote the flow associated with the system (6.1). The family of curves $S_{r}$, for $r \in(0,1]$, is defined as follows: $S_{r}:=\phi^{s}(S)$, where $s=(\omega / b) \ln r$.

\section{Step 2. Verification of properties (i)-(iv) for $S_{r}$.}

Property (i) is obvious from the construction.

To check property (ii), we will show first that the curve $S$ is transverse to the vector field given by $f$. Because the vector $\dot{q}(\lambda)$ is tangent to $M^{s}(p)$ at $q(\lambda)$, this vector is not parallel to the vector $f(q(\lambda))$. Using the fact that

$$
\Phi(t(\lambda), q(\lambda)) f(q(\lambda))=f(x(t(\lambda), q(\lambda)))
$$

it follows that the vectors $\Phi(t(\lambda), q(\lambda)) \dot{q}(\lambda)$ and $f(x(t(\lambda), q(\lambda)))$ are independent at $x(t(\lambda), q(\lambda))$. In view of this fact and the formula (12.2) for the vector $T(\lambda)$ tangent to $S$, it is clear that $f$ is everywhere transverse to $S$.

Next, for each point $Q \in S_{r}$, there exists a point $P \in S$ such that $\phi^{s}(P)=Q$. Therefore, $T_{Q} S_{r}=D \phi^{s}(P) T_{P} S$ and $f(Q)=D \phi^{s}(P) f(P)$. Since $T_{P} S$ is transverse to $f(P)$, we have that $T_{Q} S_{r}$ is transverse to $f(Q)$. This proves property (ii).

For the proof of property (iii), let us note that, due to the hyperbolicity of the orbit $\Gamma$, there exists some $C_{0}>0$ such that, for each point $x_{0} \in N$, we have $d\left(\phi^{s}\left(x_{0}\right), \Gamma\right) \leq$ $C_{0} e^{b s / \omega}$. Hence, for each $Q \in S_{r}$, if we take the point $P \in S$ such that $\phi^{s}(P)=Q$, then

$$
d(Q, \Gamma)=d\left(\phi^{s}(P), \Gamma\right) \leq C_{0} e^{\frac{b s}{\omega}}=C_{0} r,
$$

and the constant $C_{0}$ depends only on the "size" of the neighborhood $N$.

Finally, let us prove property (iv). To this end, note that for each point $Q \in S_{r}$, there is a corresponding point $P \in S$ such that $Q=\phi^{s}(P)$ and some $\lambda$ such that $P=x(t(\lambda), q(\lambda))$. Also, with an abuse of notation, let $T(Q)$ denote the vector in $T_{Q} S_{r}$ given by $T(Q)=D \phi^{s}(P) T(\lambda)$, and define $n(Q)$ to be the inward unit normal vector to $S_{r}$ at $Q$.

Using the easily verified identity $\langle f(Q), n(Q)\rangle|T(Q)|=|f(Q) \times T(Q)|$, let us note that if $r \in(0,1]$, then

$$
C_{f}^{+}(r)=\min _{Q \in S_{r}}\left\{\frac{|f(Q) \times T(Q)|}{|T(Q)|}\right\} .
$$

Also, recall formula (12.2), and note that

$$
\begin{aligned}
T(Q) & =D \phi^{s}(P) T(\lambda)=\omega \Phi(s, P) f(P)+\Phi(s, P) \Phi(t(\lambda), q(\lambda)) \dot{q}(\lambda) \\
& =\omega f(Q)+\Phi(s+t(\lambda), q(\lambda)) \dot{q}(\lambda) .
\end{aligned}
$$

Moreover, we have that $|f(Q) \times T(Q)|=|f(Q) \times \Phi(s+t(\lambda), q(\lambda)) \dot{q}(\lambda)|$. 
By an initial choice of the point $q_{1}$ sufficiently close to $p \in M^{s}(p)$, there is a number $K \geq 1$ such that

$$
\frac{1}{K} \exp \left(\int_{0}^{s+t(\lambda)} \operatorname{tr} A(\tau) d \tau\right) \leq|\Phi(s+t(\lambda), q(\lambda)) \dot{q}(\lambda)| \leq K \exp \left(\int_{0}^{s+t(\lambda)} \operatorname{tr} A(\tau) d \tau\right)
$$

where $A(t)=D f(x(t, p))$.

Let $v(Q)$ denote the unit tangent vector at $Q$ to the stable fiber through $Q$. Because $\Phi(s+t(\lambda), q(\lambda)) \dot{q}(\lambda)$ is tangent to the stable fiber through $Q$, we have that

$$
\begin{aligned}
|f(Q) \times T(Q)| & =|f(Q) \times \Phi(s+t(\lambda), q(\lambda)) \dot{q}(\lambda)| \\
& \geq \frac{1}{K}|f(Q) \times v(Q)| \exp \left(\int_{0}^{s+t(\lambda)} \operatorname{tr} A(\tau) d \tau\right),
\end{aligned}
$$

and there is a constant $C_{2}>0$ such that

$$
\begin{aligned}
|T(Q)| & \leq \omega|f(Q)|+|\Phi(s+t(\lambda), q(\lambda)) \dot{q}(\lambda)| \\
& \leq \omega|f(Q)|+K \exp \left(\int_{0}^{s+t(\lambda)} \operatorname{tr} A(\tau) d \tau\right) \\
& \leq \omega|f(Q)|+C_{2} .
\end{aligned}
$$

Therefore, using the above estimates and the fact that the quantities $|f|$ and $\mid f(Q) \times$ $v(Q) \mid$ are bounded below over $N$, there is a constant $C_{3}>0$ such that

$$
\begin{aligned}
\frac{|f(Q) \times T(Q)|}{|T(Q)|} & \geq \frac{|f(Q) \times v(Q)|}{K\left(\omega|f(Q)|+C_{2}\right)} \exp \left(\int_{0}^{s+t(\lambda)} \operatorname{tr} A(\tau) d \tau\right) \\
& \geq C_{3} \exp \left(\int_{0}^{s} \operatorname{tr} A(\tau) d \tau\right) .
\end{aligned}
$$

If $m$ is a nonnegative integer and $0 \leq \lambda<\omega$ is such that $s=m \omega+\lambda$, then there are constants $C_{1}>0$ and $C_{4}>0$ such that

$$
\begin{aligned}
C_{3} \exp \left(\int_{0}^{s} \operatorname{tr} A(\tau) d \tau\right) & =C_{3} \exp \left(\int_{0}^{m \omega+\lambda} \operatorname{tr} A(\tau) d \tau\right) \\
& \geq C_{4} \exp \left(\int_{0}^{m \omega} \operatorname{tr} A(\tau) d \tau\right) \\
& =C_{4} e^{b m}=C_{4} e^{\frac{b s-b \lambda}{\omega}} \geq C_{1} r .
\end{aligned}
$$

Thus, we have proved that $C_{f}(r) \geq C_{1} r$.

Proof of Proposition 6.2. If the families of curves $S_{r}^{+}$and $S_{r}^{-}$are "suspended" to tori in the space $\mathbb{R}^{2} \times \mathbb{R}$, then the corresponding tori can be shown to satisfy conditions analogous to the conditions (i)-(iv) that are defined in the proof of Proposition 6.1. The verification of each condition is essentially identical to the corresponding proof in Proposition 6.1; we omit the details.

Acknowledgments. Finally, we thank the referees for carefully reading the original version of this paper. Their comments led to many improvements. 


\section{REFERENCES}

[1] N. N. Bogoliubov And Y. A. Mitropolsky, The method of integral manifolds in nonlinear mechanics, Contrib. Differential Equations, 2 (1963), pp. 123-196.

[2] C. Chicone, Invariant tori for periodically perturbed oscillators, Publ. Mat., 41 (1997), pp. $57-83$.

[3] N. Fenichel, Persistence and smoothness of invariant manifolds for flows, Indiana Univ. Math. J., 21 (1971), pp. 193-226.

[4] N. Fenichel, Geometric singular perturbation theory for ordinary differential equations, J. Differential Equations, 31 (1979), pp. 53-98.

[5] J. Guckenheimer and P. Holmes, Nonlinear Oscillations, Dynamical Systems, and Bifurcations of Vector Fields, 2nd ed., Springer-Verlag, New York, 1986.

[6] J. Hale, Ordinary Differential Equations, Robert E. Krieger Pub. Co. Inc., Huntington, NY, 1980.

[7] M. Hirsch, C. C. Pugh, ANd M. Shub, Invariant Manifolds, Lecture Notes in Math., vol. 583, Springer-Verlag, New York, 1977.

[8] N. KopelL, Invariant manifolds and the initialization problem for some atmospheric equations, Phys. D, 14 (1985), pp. 203-215.

[9] V. K. Melnikov, On the stability of the center for time-periodic perturbations, Trans. Moscow Math. Soc., 12 (1963), pp. 1-56.

[10] C. Robinson And J. MURDock, Some mathematical aspects of spin-orbit resonance. II. Celestial Mech., 24 (1981), 83-107.

[11] S. Wiggins, Introduction to Applied Nonlinear Dynamical Systems and Chaos, SpringerVerlag, New York, 1990.

[12] S. Wiggins, Normally Hyperbolic Invariant Manifolds in Dynamical Systems, Springer-Verlag, New York, 1994. 